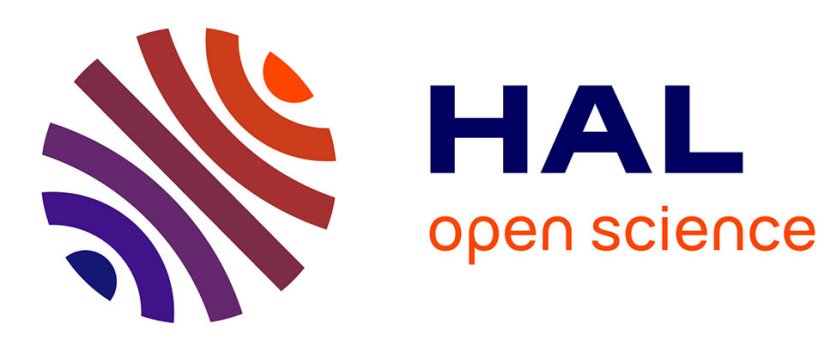

\title{
Beyond the limitation of Geometrical Shock Dynamics for diffraction over wedges
}

Julien Ridoux, Nicolas Lardjane, Laurent Monasse, François Coulouvrat

\section{To cite this version:}

Julien Ridoux, Nicolas Lardjane, Laurent Monasse, François Coulouvrat. Beyond the limitation of Geometrical Shock Dynamics for diffraction over wedges. 2018. hal-01880213

\section{HAL Id: hal-01880213 https://hal.science/hal-01880213}

Preprint submitted on 24 Sep 2018

HAL is a multi-disciplinary open access archive for the deposit and dissemination of scientific research documents, whether they are published or not. The documents may come from teaching and research institutions in France or abroad, or from public or private research centers.
L'archive ouverte pluridisciplinaire HAL, est destinée au dépôt et à la diffusion de documents scientifiques de niveau recherche, publiés ou non, émanant des établissements d'enseignement et de recherche français ou étrangers, des laboratoires publics ou privés. 


\title{
Beyond the limitation of Geometrical Shock Dynamics for diffraction over wedges
}

\author{
J. Ridoux ${ }^{1,3}$, N. Lardjane ${ }^{1}$, L. Monasse ${ }^{2}$, and F. Coulouvrat ${ }^{3}$ \\ ${ }^{1}$ CEA, DAM, DIF, Arpajon F-91297, France. nicolas.lardjane@cea.fr \\ ${ }^{2}$ Université Côte d'Azur, Inria, CNRS, LJAD, EPC COFFEE, Parc Valrose, 06108 Nice, France. \\ ${ }^{3}$ Institut Jean Le Rond d'Alembert, UMR 7190 CNRS, Sorbonne Université, Paris, France.
}

September 24, 2018

\begin{abstract}
Geometrical Shock Dynamics (GSD) is a simplified model for nonlinear shock wave propagation for which the front evolution is governed by a local relation between the geometry of the shock and its velocity, so-called $A-M$ rule. Numerous studies have proven the ability of the GSD model to estimate correctly the leading shock front in interaction with obstacles. Nevertheless, a solution for the problem of diffraction over a convex corner does not always exist, especially for weak shocks. To overcome this limitation, we propose an $a d-h o c$ modification of the $A-M$ relation for two-dimensional configurations: an extra term based on the transverse variation of the Mach number is added. This new closure is fitted against experimental observations, which ensures, by construction, a correct behaviour for expansive shocks. A Lagrangian numerical solver is developed, for which this new term is activated only on specific parts of the front. Results of this new model are compared with the original GSD model, experiments, and Eulerian simulations for several cases of increasing complexity. A noticeable improvement of the solution is observed.
\end{abstract}

Keywords. Shock wave, Geometrical Shock Dynamics, Shock diffraction, Lagrangian scheme

\section{Introduction}

The problem of determining the motion of shock waves has received considerable attention over the last several decades. The development of simplified models, able to estimate the position, shape, and strength of a shock, is of prime importance in several domains such as the pyrotechnics industry, explosion hazards, or noise annoyance among others. In 1957, 
Whitham published a hyperbolic model called Geometrical Shock Dynamics (GSD) [32, 33, 34] able to estimate at a moderate cost, but with reasonable accuracy, the propagation of a shock interacting with geometrical elements. This model is based on the decomposition of the shock front into elementary ray tubes. Assuming small changes in the ray tube area and neglecting the influence of the post-shock flow on the shock, a simple relation linking the local curvature and velocity of the front, known as the $A-M$ rule, is obtained [4].

It is well known that GSD is quite accurate for sustained shock propagation problems. It has been investigated by numerous authors in the past, for cases of converging flows [25, 24], propagation through nonuniform media [5], but also for outdoor propagation [3] among others. Nevertheless, the model suffers from a limitation for the problem of shock diffraction over a convex wall. For sufficiently weak shocks, no solution of GSD model exists up to the wall above a given deflection angle. This is in contradiction with experimental studies [29] showing that the diffracted shock front should still exist at the wall, even for weak shocks and at large deflection angles. Some modifications of GSD, such as its extension to post-shock flow [4], or another treatment of the wall condition [1], are able to recover the inflection point experimentally observed for strong shocks, but do not remove the limitation. The more recent Kinematic model [26, 27, 16], is no more efficient to remove this restriction [18]. Oshima et al. [15] studied several extensions of Whitham's $A-M$ rule to improve the GSD model. They concluded that all these modifications, based on one-dimensional flow analysis, are unable to reproduce the experimental behaviour for the diffraction of a weak shock over a convex wall. These authors suggested that this difference could be caused by a kind of interaction between ray tubes. He thus proposed a correction of the $A-M$ relation by taking into account the shear stress in the Euler equations holding in each ray tube, with a closure similar to a turbulent viscosity. Although no physical reason justifies this approach, the gap between a linearized solution from this theory and experiments is reduced for incident Mach numbers ranging from 1.5 to 2.8. Another derivation with the only assumption of interaction between ray tubes is presented in [14]. In both cases, the modified $A-M$ rule involves complex terms describing some transverse variation along the shock curve. The relation seems difficult to solve and requires an in-depth study which has never been carried out. Moreover, there is no indication that the restriction is removed.

From a numerical point of view, many algorithms have been developed for the GSD model. They are based on front-tracking methods [7, 20], Eulerian conservative schemes [22, 23], level set methods [31], or a fast-marching like approach [12].

The objective of this paper is to present an ad-hoc extension of the GSD model, much simpler than Oshima's one, and designed to systematically remove the limitation of the model. For this purpose, the derivation of the GSD model in a two-dimensional configuration is first reviewed in section 2. Using experimental data, we give in section 3 an empirical law for the planar shock diffraction over a convex corner under the assumption that the solution is self-similar. We then propose a modification of the $A-M$ rule by modeling a transverse flow with this law. Following [7], but with a different integration method for the $A-M$ relation, a 2D conservative Lagrangian scheme is developed and validated in section 4 . Finally, results 
are discussed in section 5 .

\section{Problem set up}

In this paper, we consider the propagation of a two-dimensional shock wave in a uniform, quiescent, and calorically perfect gas. We denote by $\rho, p$, and $v$ the density, pressure, and fluid velocity respectively. For a perfect gas, the speed of sound $c$ reads

$$
c=\sqrt{\frac{\gamma p}{\rho}},
$$

with $\gamma$ the specific heat ratio of the gas, supposed constant and equal to 1.4 for air. These variables are indexed by 0 for the initial state of the gas at rest.

We introduce the shock Mach number:

$$
M=\frac{U}{c_{0}} \geq 1
$$

where $U$ denotes the shock velocity. The shock position, $\boldsymbol{x}$, is then a solution of the Ordinary Differential Equation (ODE):

$$
\frac{\mathrm{d} \boldsymbol{x}}{\mathrm{d} t}=U \boldsymbol{n}=c_{0} M \boldsymbol{n},
$$

where $t$ is the arrival time of the shock, and $\boldsymbol{n}$ is the unit outward normal vector at the front.

\subsection{Geometrical Shock Dynamics model in a two-dimensional con- figuration}

In this section, we recall the classical derivation of the Geometrical Shock Dynamics (GSD) model in two dimension (2D) [32], which will be useful for our model extension.

The key idea behind the GSD model consists in splitting the shock front into elementary areas, $A$, propagating along ray tubes in which the cross-flow is neglected. The model of shock propagation is thus reduced to the $1 \mathrm{D}$ problem of a planar shock moving into a channel with varying cross section.

The successive positions of the shock are given by a set of curves $\alpha=$ cst, shown as full lines in figure 1. The orthogonal trajectories of this set of curves, called rays, are represented by curves $\beta=$ cst and are drawn as dashed lines. The coordinate $\alpha$ and the arrival time of the front, $t$, are related by $\alpha=c_{0} t$ for a single pass front. Since $c_{0}$ is constant, $\alpha$ is also called a pseudo-time for the sake of simplicity. Geometrical relations in the local coordinates of the shock, $(\alpha, \beta)$, are straightforward [32]:

$$
\left\{\begin{array}{l}
\frac{\partial \theta}{\partial \beta}-\frac{1}{M} \frac{\partial A}{\partial \alpha}=0 \\
\frac{\partial \theta}{\partial \alpha}+\frac{1}{A} \frac{\partial M}{\partial \beta}=0
\end{array}\right.
$$




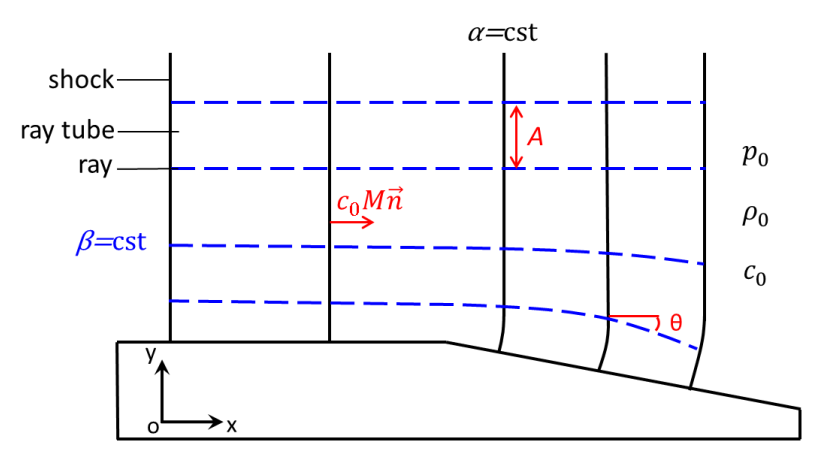

Figure 1: 2D shock wave propagation in the Geometrical Shock Dynamics theory. The solid black lines are the successive shock positions and correspond to $\alpha$ coordinates. The dashed blue lines are the rays and correspond to $\beta$ coordinates. $\theta$ is the direct angle between the $(O x)$ axis and rays. Rays delimit ray tubes of area $A$.

where $\theta$ is the direct angle between the $(O x)$ axis and rays. Two neighbouring rays delimit a ray tube of cross-sectional area $A$. It is worth noticing that, since $A$ measures the distance between rays, the curvilinear abscissa along the shock, $s$, is determined by the differential relation: $\mathrm{d} s=A \mathrm{~d} \beta$. By considering a ray tube as a channel with rigid walls, a simple law linking $A$ to $M$ closes the system. This relation, called $A-M$ rule, is obtained from the 1D Euler system with varying cross-section [4]:

$$
\frac{1}{A} \frac{\mathrm{d} A}{\mathrm{~d} \alpha}+\frac{M \lambda(M)}{M^{2}-1} \frac{\mathrm{d} M}{\mathrm{~d} \alpha}+h(M) Q=0
$$

where

$$
\begin{gathered}
\lambda(M)=\left(1+\frac{2}{\gamma+1} \frac{1-\mu^{2}}{\mu}\right)\left(1+2 \mu+\frac{1}{M^{2}}\right), \\
h(M)=\frac{\gamma+1}{2} \frac{\mu(\mu-1)}{M^{2}-1}
\end{gathered}
$$

and $\mu$ is the post-shock Mach number:

$$
\mu=\sqrt{\frac{(\gamma-1) M^{2}+2}{2 \gamma M^{2}+1-\gamma}} .
$$

The term $Q=\frac{\left(\partial_{t} p+\rho c \partial_{t} v\right)_{-}}{p_{0} c_{0}^{3}}$, indexed by - for quantities just behind the shock, contains all the post-shock flow terms. $Q$ is an unknown of the problem. As

$$
\lim _{M \rightarrow 1} h(M)=-0.6 \quad \text { and } \quad \lim _{M \rightarrow+\infty} h(M)=0,
$$

Whitham chose to neglect this term under the assumption of the smallness of post-shock effects $Q$. The resulting equation,

$$
\frac{1}{A} \frac{\mathrm{d} A}{\mathrm{~d} \alpha}+\frac{M \lambda(M)}{M^{2}-1} \frac{\mathrm{d} M}{\mathrm{~d} \alpha}=0,
$$


appears to work remarkably well in a large number of configurations $[34,7,18]$. Finally, the GSD model is composed of the geometrical system (2) and the $A-M$ relation (4).

The function $\lambda$ is bounded and increasing from 4 at $M=1$ to

$$
\lambda_{\infty}=1+\frac{2}{\gamma}+\sqrt{\frac{2 \gamma}{\gamma-1}} \approx 5.074, \quad \text { for } \gamma=1.4
$$

as $M \rightarrow+\infty$.

From (4), $A$ depends only on $M$ and can be considered as dimensionless:

$$
A(M)=\exp \left(-\int_{M_{0}}^{M} \frac{m \lambda(m)}{m^{2}-1} \mathrm{~d} m\right)
$$

with $M_{0}$ a reference Mach number. It is straightforward to prove the hyperbolicity of the system (2) provided that $A^{\prime}(M)<0$, which is verified as $\lambda(M)>0$ and $M \geq 1$. Some waves may thus develop on the shock front. They are responsible for the modification of the intensity, shape, and orientation along with front evolution. In particular, discontinuities, called shock-shocks, appear. They correspond to the triple point position on the shock when a Mach stem arises. These perturbations travel at velocity

$$
\frac{A \mathrm{~d} \beta}{\mathrm{d} \alpha}= \pm A u(M)
$$

with

$$
A u(M)=A(M) u(M)=\sqrt{\frac{M^{2}-1}{\lambda(M)}} .
$$

The characteristic form of the GSD system (2) is expressed as:

$$
\left\{\frac{\partial}{\partial \alpha} \pm u(M) \frac{\partial}{\partial \beta}\right\}(\theta \pm \omega(M))=0
$$

where

$$
\omega(M)=\int_{1}^{M} \frac{\mathrm{d} m}{A u(m)}=\int_{1}^{M} \sqrt{\frac{\lambda(m)}{m^{2}-1}} \mathrm{~d} m<+\infty .
$$

Finally, relations (9) provide the Riemann invariants:

$$
\theta \pm \omega(M)=\text { cst, on } C^{ \pm}: \frac{\mathrm{d} \beta}{\mathrm{d} \alpha}= \pm u(M)
$$

The Cartesian coordinates $(x, y)$ can be expressed from the local coordinates $(\alpha, \beta)$ :

$$
\begin{array}{ll}
\frac{\partial x}{\partial \alpha}=M \cos \theta \quad ; \quad \frac{\partial y}{\partial \alpha}=M \sin \theta \\
\frac{\partial x}{\partial \beta}=-A \sin \theta \quad ; \quad \frac{\partial y}{\partial \beta}=A \cos \theta .
\end{array}
$$


Considering (11) and (7), one has along the characteristics $C^{ \pm}$:

$$
\left\{\begin{array}{l}
\frac{\mathrm{d} x}{\mathrm{~d} \alpha}=M \cos \theta \mp A u(M) \sin \theta \\
\frac{\mathrm{d} y}{\mathrm{~d} \alpha}=M \sin \theta \pm A u(M) \cos \theta .
\end{array}\right.
$$

The characteristics $C^{ \pm}$can be rewritten as

$$
C^{ \pm}: \frac{\mathrm{d} y}{\mathrm{~d} x}=\tan (\theta \pm \nu), \text { with } \tan (\nu)=\frac{A u(M)}{M} .
$$

In the presence of an obstacle, wall boundaries coincide with rays in Whitham's theory. Consequently, the front is orthogonal to the wall.

\subsection{Radial solutions for GSD}

For a planar $(d=1)$, cylindrical $(d=2)$ or spherical $(d=3)$ shock wave, the solution depends only on the radial coordinate $r$. The Mach number, $M$, and the arrival time, $\alpha$, are solution of the one-dimensional ODE:

$$
\left\{\begin{array}{l}
\frac{\mathrm{d} \alpha(r)}{\mathrm{d} r}=\frac{1}{M(r)} \\
\frac{\mathrm{d} M(r)}{\mathrm{d} r}=-\frac{M^{2}(r)-1}{M(r) \lambda(M(r))} \frac{d-1}{r} .
\end{array}\right.
$$

For a given initial condition, $\alpha\left(r_{0}\right)=\alpha_{0}$ and $M\left(r_{0}\right)=M_{0}$ where $r_{0}$ is the initial shock position, this system can be solved numerically with a high-order algorithm. However, analytical solutions are easily obtained in the strong shock limit $(M \gg 1)$ :

$$
\mid \begin{aligned}
& \alpha_{a}(r)=\alpha_{0}+\frac{r_{0}}{M_{0}\left(\frac{d-1}{\lambda \infty}+1\right)}\left(\left(\frac{r}{r_{0}}\right)^{\frac{d-1}{\lambda \infty}+1}-1\right) \\
& M_{a}(r)=M_{0}\left(\frac{r_{0}}{r}\right)^{\frac{d-1}{\lambda \infty}}
\end{aligned}
$$

where $\lambda_{\infty}$ is given by (5). These solutions can be expressed in terms of $\alpha$ :

$$
\begin{aligned}
& r_{a}(\alpha)=r_{0}\left(\left(\alpha-\alpha_{0}\right) \frac{M_{0}}{r_{0}}\left(\frac{d-1}{\lambda_{\infty}}+1\right)+1\right)^{1 /\left(\frac{d-1}{\lambda_{\infty}}+1\right)} \\
& M_{a}(\alpha)=M_{0}\left(\frac{r_{0}}{r(\alpha)}\right)^{\frac{d-1}{\lambda_{\infty}}},
\end{aligned}
$$

which is helpful for the mesh convergence studies reported in section 4.5. 


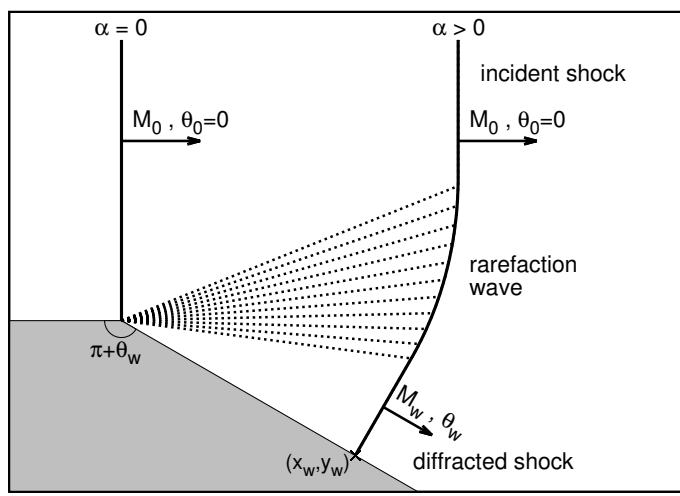

(a)

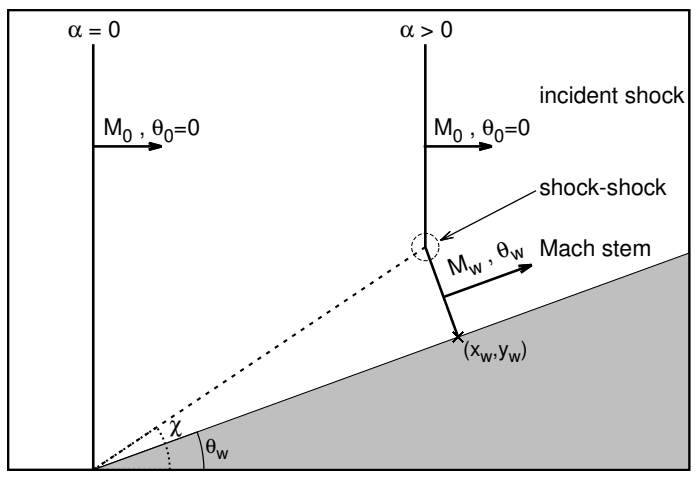

(b)

Figure 2: Scheme of a planar shock diffraction over a convex corner (Fig. (a), expansion wave) and over a concave corner (Fig. (b), shock-shock occurrence). The deflection angle of the corner is denoted $\theta_{\mathrm{w}}$.

\subsection{Riemann problem}

The elementary Riemann problem corresponds to the diffraction of a planar shock over a corner as illustrated in figure 2. The shock travels from the left to the right at an initial Mach number $M_{0}$ and diffracts over the corner at time $\alpha=0$. The first state for the Riemann problem is given by the incident shock: $\left(M_{0}, \theta_{0}=0\right)$. After diffraction, the wall Mach number, $M_{\mathrm{w}}$, and the deflection angle, $\theta_{\mathrm{w}}$, represent the second state. As these quantities are independent of $\alpha$, the point of interaction between the shock and the wall is given by

$$
x_{\mathrm{w}}=x_{0}+\alpha M_{\mathrm{w}} \cos \theta_{\mathrm{w}}, \quad y_{\mathrm{w}}=y_{0}+\alpha M_{\mathrm{w}} \sin \theta_{\mathrm{w}},
$$

using (11a), where $\left(x_{0}, y_{0}\right)=(0,0)$ is the coordinate of the corner. For a convex corner, a simple wave links the states $\left(M_{0}, \theta_{0}\right)$ to $\left(M_{\mathrm{w}}, \theta_{\mathrm{w}}\right)$, while, for a concave corner, a shock-shock appears on the shock front. These problems are well detailed in [34, 12].

Continuous case. For the convex corner case, all $C^{-}$characteristics cross the initial shock. The solution is a simple wave and is self-similar [34]. The shock front is then split in three parts (see Fig. 2(a)) from top to bottom:

- the unperturbed, incident straight shock;

- then a smooth transition under the form of a curved shock front, which plays here the role of an expansion fan for the Riemann problem of the GSD model;

- and finally a straight shock, orthogonal to the wall, with a constant Mach number $M_{\mathrm{w}}$. 
The rarefaction fan takes its origin at the corner. On each $C^{+}$characteristic of the fan, the Mach number, $M$, and the orientation of the ray, $\theta$, are linked by the differential expression (9):

$$
\frac{\mathrm{d} \theta}{\mathrm{d} \alpha}=\sqrt{\frac{\lambda(M)}{M^{2}-1}} \frac{\mathrm{d} M}{\mathrm{~d} \alpha} \text { on } C^{+}: \frac{\mathrm{d} y}{\mathrm{~d} x}=\tan (\theta+\nu) .
$$

By integrating (17) from the initial time $\alpha=0$, where $\theta=\theta_{0}=0$ and $M=M_{0}$, to time $\alpha$, one gets:

$$
\theta-\theta_{0}=\omega(M)-\omega\left(M_{0}\right)=\int_{M_{0}}^{M} \frac{\mathrm{d} m}{A u(m)} .
$$

Finally, as $\theta$ and $M$ are constant along each characteristic, the solution can be expressed as:

$$
\begin{array}{ll}
M=M_{0} & \text { for } \theta=\theta_{0} \\
\int_{M_{0}}^{M} \frac{\mathrm{d} m}{A u(m)}=\theta-\theta_{0} & \text { for } \theta_{\mathrm{w}} \leq \theta \leq \theta_{0} \\
M=M_{\mathrm{w}} & \text { for } \theta=\theta_{\mathrm{w}},
\end{array}
$$

where (8) gives the expression of $A u$. From (19) and (12), the form of the shock in the rarefaction fan at pseudo-time $\alpha$ is determined by

$$
\mid \begin{aligned}
& x=x_{0}+\alpha(M \cos \theta \mp A u(M) \sin \theta) \\
& y=y_{0}+\alpha(M \sin \theta \pm A u(M) \cos \theta),
\end{aligned}
$$

for $\theta_{\mathrm{w}} \leq \theta \leq \theta_{0}$. For example, we present in figure 3 the solutions at $\alpha=1$ for $M_{0}=1.5$ and several deflection angles, $\theta_{\mathrm{w}}$, obtained with (19)-(20). The integral in (19b) is approximated with a Simpson method. For each $\theta$ in the set $\left\{\theta_{i} \in\left[\theta_{\mathrm{w}}, \theta_{0}\right], i=1, \ldots, N\right\}$, the nonlinear equation is solved with a Newton algorithm. The relation (13) gives the first and last characteristics with $(M, \theta)=\left(M_{0}, \theta_{0}\right)$ and $(M, \theta)=\left(M_{\mathrm{w}}, \theta_{\mathrm{w}}\right)$ respectively. As the solution is self-similar, we observe that the shock fronts describe the same curve for any $\theta_{\mathrm{w}}$. This property will be used later on in this paper.

Shock-shock occurrence. For a concave corner, a shock-shock immediately appears on the shock front as sketched in figure 2(b). Corresponding Rankine-Hugoniot relations must be developed in consequence [34]. We denote $\chi$ the direct angle between the $(O x)$ axis and 


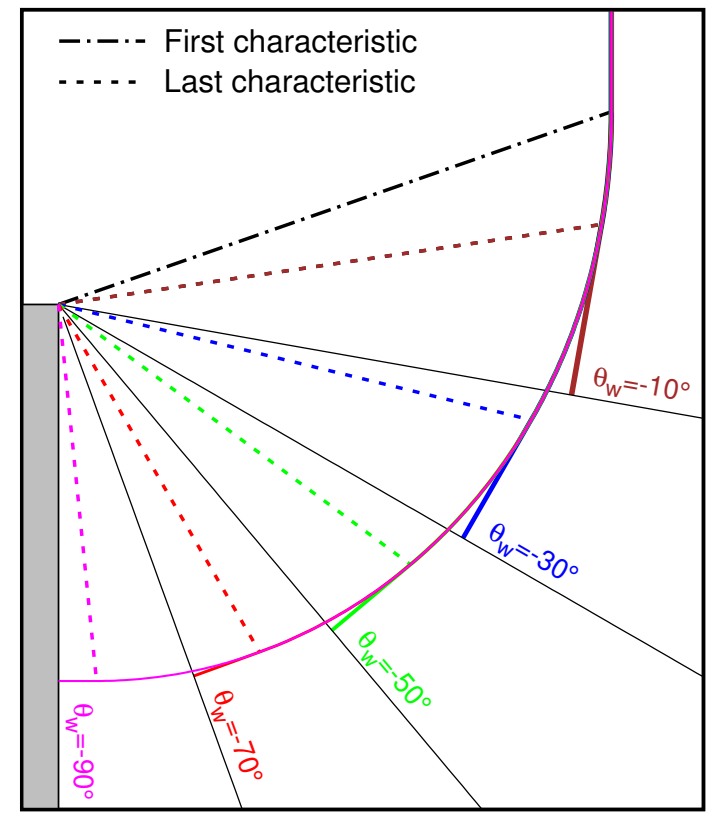

Figure 3: Diffraction of a planar shock at Mach number 1.5 over a convex corner of angle $\theta_{\mathrm{w}}<0$. Color solid lines: shock front position for the GSD model (eqs. (19), (20) and (13)) at $\alpha=1$ for different deflection angles, $\theta_{\mathrm{w}}$. Dash-dotted line: first characteristic (identical for all angles). Dashed color lines: last characteristic of the expansion fan (same color code as for shock fronts). Thin black lines: rigid wall position. Value of deflection angle is reported on the figure with same color code as shock front.

the shock-shock trajectory. The solution $\left(M_{\mathrm{w}}, \chi\right)$ is given implicitly by

$$
\left\{\begin{array}{l}
\tan \left(\theta_{\mathrm{w}}\right)=\frac{\sqrt{\left(\left(\frac{M_{\mathrm{w}}}{M_{0}}\right)^{2}-1\right)\left(1-\left(\frac{A_{\mathrm{w}}}{A_{0}}\right)^{2}\right)}}{1+\frac{A_{\mathrm{w}}}{A_{0}} \frac{M_{\mathrm{w}}}{M_{0}}} \\
\tan \left(\chi-\theta_{\mathrm{w}}\right)=\frac{A_{\mathrm{w}}}{A_{0}} \sqrt{\frac{1-\left(\frac{M_{0}}{M_{\mathrm{w}}}\right)^{2}}{1-\left(\frac{A_{\mathrm{w}}}{A_{0}}\right)^{2}}}
\end{array}\right.
$$

where $\frac{A_{\mathrm{w}}}{A_{0}}=A\left(M_{\mathrm{w}}\right)$ is defined by $(6) . M_{\mathrm{w}}$ is obtained from the first relation by using a Newton algorithm, $\chi$ is then calculated with the second one.

\subsection{Limitation of the GSD model}

In the case of diffraction over a convex corner, $\theta-\theta_{0}<0$. Then, from (18), the Mach number decreases along the shock from $M_{0}$ to $M_{\mathrm{w}}$, the value at the wall. Since $\theta_{\mathrm{w}}-\theta_{0}$ and 
$M_{0}$ are known, the wall Mach number is calculated with the expression holding on the last characteristic:

$$
\int_{M_{0}}^{M_{\mathrm{w}}} \sqrt{\frac{\lambda(m)}{m^{2}-1}} \mathrm{~d} m=\theta_{\mathrm{w}}-\theta_{0}<0 .
$$

From (22), one can easily show that a solution such that $M_{\mathrm{w}} \geq 1$ exists if and only if $M_{0} \geq M_{\lim }>1$, with $M_{\text {lim }}$ the minimal reachable value of the incident Mach number:

$$
\int_{1}^{M_{\mathrm{lim}}} \sqrt{\frac{\lambda(m)}{m^{2}-1}} \mathrm{~d} m=-\theta_{\mathrm{w} .}
$$

Let us remark that this problem is not encountered for the compressive problem. Indeed, in this case we have $M_{\mathrm{w}} \geq M_{0} \geq 1$ and (21a) always provides a solution according to the intermediate value theorem.

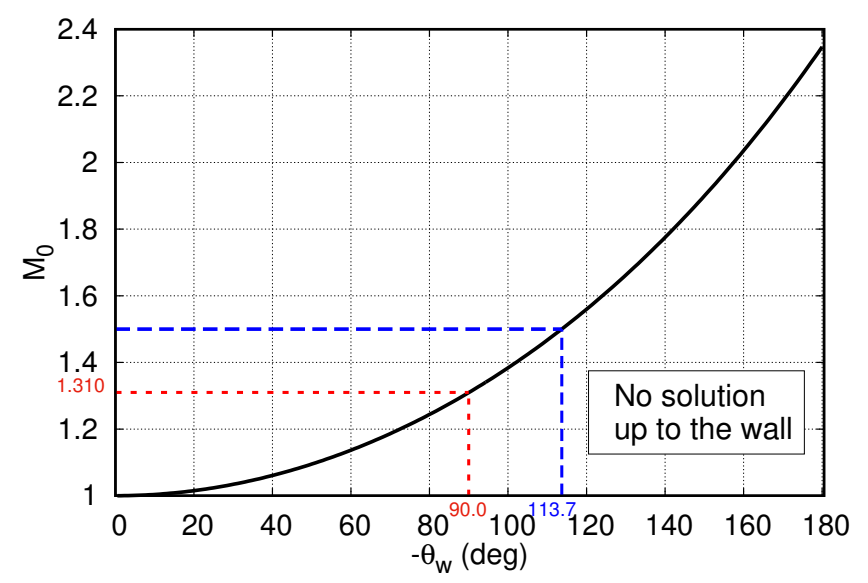

Figure 4: Minimal value for the initial Mach number, $M_{0}$, with respect to $\theta_{\mathrm{w}}$ in order for a solution to exist for the planar shock diffraction problem over a convex wall (relation (23)). There is no solution up to the wall if $\left(M_{0}, \theta_{\mathrm{w}}\right)$ is strictly below the curve. Two particular cases discussed in text are indicated as examples by red and blue dotted lines.

The restriction (23) is plotted in figure 4 . For any pair of variables $\left(M_{0}, \theta_{\mathrm{w}}\right)$ in the area below the curve, there is no solution up to the wall for the diffraction of a planar shock over a convex corner. For instance, we have $M_{\text {lim }} \approx 1.310$ for $\theta_{\mathrm{w}}=-\pi / 2$, and if $M_{0}=1.1$, the solution exists up to a deflection angle of approximately $-49^{\circ}$ as shown in figure 5 .

Skews [29] has shown experimentally that, contrary to the GSD prediction, the diffracted shock front still exists at the wall, even for weak shocks $\left(M_{0}=1.2\right)$ and at large deflection angles $\left(\theta_{\mathrm{w}}\right.$ up to $\left.-165^{\circ}\right)$, as shown in figure 6 . Indeed, for $M_{0}=1.5$ for example, we see that GSD does not have a solution for $\theta_{\mathrm{w}}<-113.7^{\circ}$, whereas experiments show that the wall Mach number $M_{\mathrm{w}}$ keeps larger than unity up to $-150^{\circ}$ at least. The extension of the GSD model with post-shock flow effects [4] does not seem to correct this default as $M_{\mathrm{w}}$ is further reduced in this case in comparison to the classical GSD form. Alternative approaches, such 


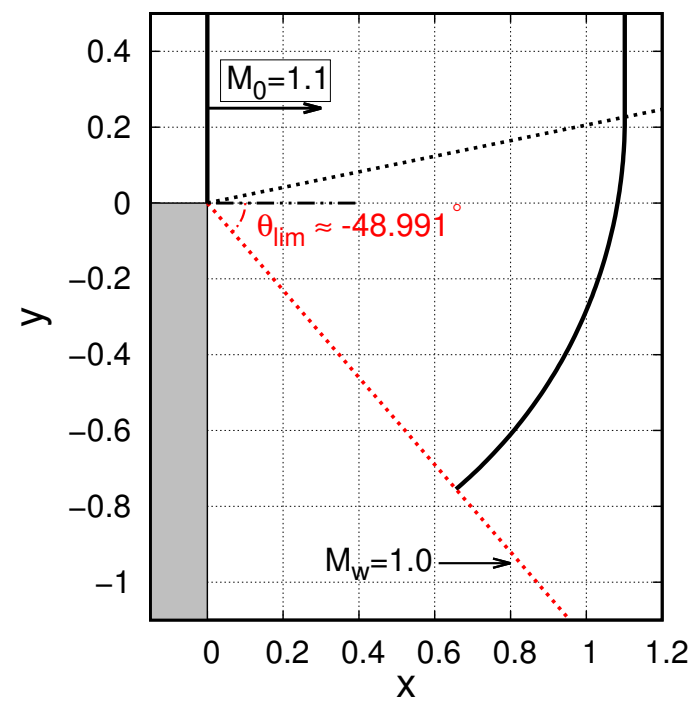

Figure 5: Shock position for the GSD model for the planar shock diffraction of a shock at $M_{0}=1.1$ over a convex corner at $\theta_{\mathrm{w}}=-\pi / 2$ (eqs. (19)-(20)). The GSD model has no solution in the area between the wall and the red dotted line.

as the Kinematic model [18], or theoretical modifications of the $A-M$ relation [15, 14], do not seem much more efficient to remove this restriction.

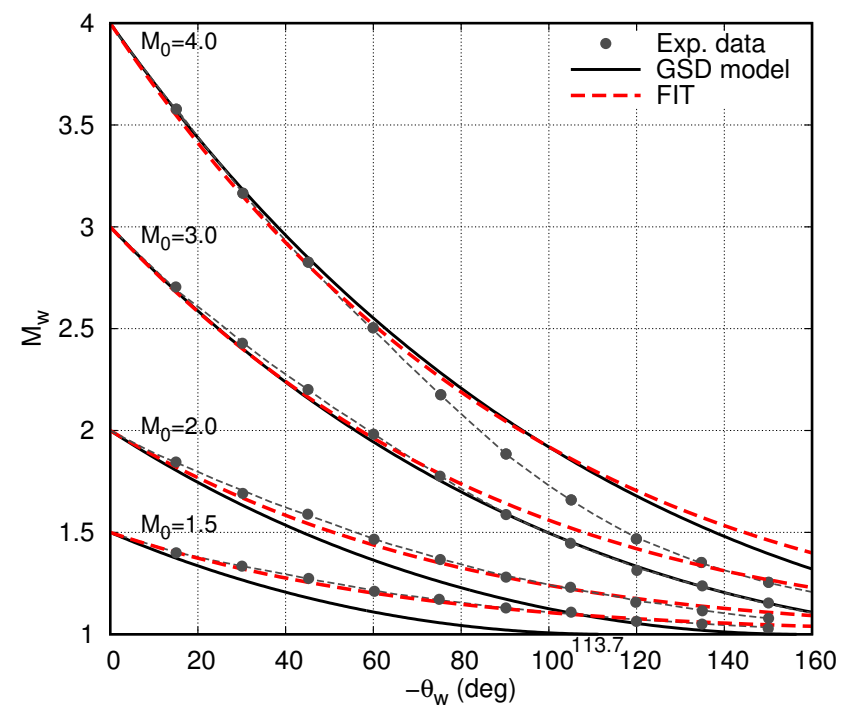

Figure 6: Diffraction of a planar shock over a convex corner. Wall Mach number, $M_{\mathrm{w}}$, with respect to deflection angle, $\theta_{\mathrm{w}}$ in degrees, for different incident Mach numbers $M_{0}$. Comparison between experimental data [29] (black dots), the GSD model (22) (black solid line), and our analytical fit (24)-(25) (red dashed line). 


\section{Taking into account transverse flow in Whitham's $A-M$ rule}

Oshima et al. $[15,14]$ proposed a generalized theory of GSD which includes shear effects. The correction of the $A-M$ relation suggests a kind of interaction between ray tubes which transposes into a transverse variation along the shock curve. The study of this extension is limited to only a linearized solution which shows some improvement of the solution. Indeed, the correction increases the wall Mach number, which in the range from $M_{0}=1.5$ to 2.8 , is in better agreement with experiment. Nevertheless, the modified $A-M$ relation is complex to solve in general and there is no indication that the limitation is removed. Furthermore, the transverse terms will modify the behaviour of the model for compressive waves (concave corner), which may lower the quality of the model.

Here, we propose a simpler closure than previous works [15, 14], based on the experiments of Skews [29]. It is designed so as to remove the limitation of the GSD model for all expansive shocks and do not affect compressive parts.

\subsection{Empirical law for the rarefaction fan}

For a given deflection angle $\theta_{\mathrm{w}}$, Skews' experimental data [29], presented in figure 6, can be gathered along curves starting from the point $\left(M_{0}, M_{\mathrm{w}}\right)=(1,1)$ and having the expression:

$$
\frac{M_{\mathrm{w}}^{2}-1}{M_{0}^{2}-1}=p\left(\theta_{\mathrm{w}}\right), \quad \theta_{\mathrm{w}}<0 \text { in } \mathrm{rad},
$$

as shown in figure $7(a)$, or equivalently

$$
M_{\mathrm{w}}\left(\theta_{\mathrm{w}}, M_{0}\right)=\sqrt{1+p\left(\theta_{\mathrm{w}}\right)\left(M_{0}^{2}-1\right)} .
$$

We define an analytical approximation for $p$ and use a nonlinear regression to obtain

$$
p\left(\theta_{\mathrm{w}}\right)=\exp \left(k \theta_{\mathrm{w}}\right), \quad \text { with } k=0.985 .
$$

The choice of this simple form will be explained in section 3.2. The fit $p\left(\theta_{\mathrm{w}}\right)$ is plotted in figure 7(b) and leads to only a slight difference with calculated slopes. The expression (24)(25) is drawn in figure 6. A much better agreement with experimental data is found for $M_{0} \leq 2$ and $-\theta_{w}<60^{\circ}$ in comparison to GSD. An overestimation of the wall Mach number is observed for strong shocks $\left(M_{0} \geq 2\right)$ and large deflection angles $\left(-\theta_{w}>80^{\circ}\right)$, nevertheless results appear acceptable in regard to the difference between the GSD model and Skews's data. In this configuration, the law (24)-(25) predicts a wall Mach number with the same order of accuracy as Whitham's theory.

Function (25) is established from experimental data between $1.5 \leq M_{0} \leq 4$ and $-140^{\circ} \leq$ $\theta_{\mathrm{w}} \leq 0$, but is used for all $\theta_{\mathrm{w}} \leq 0$ and $M_{0} \geq 1$ in practice.

From a modelling point of view, it is desirable that the wall Mach number, and a fortiori the last characteristic of the rarefaction fan, verify the law (24)-(25). Since the GSD solution 


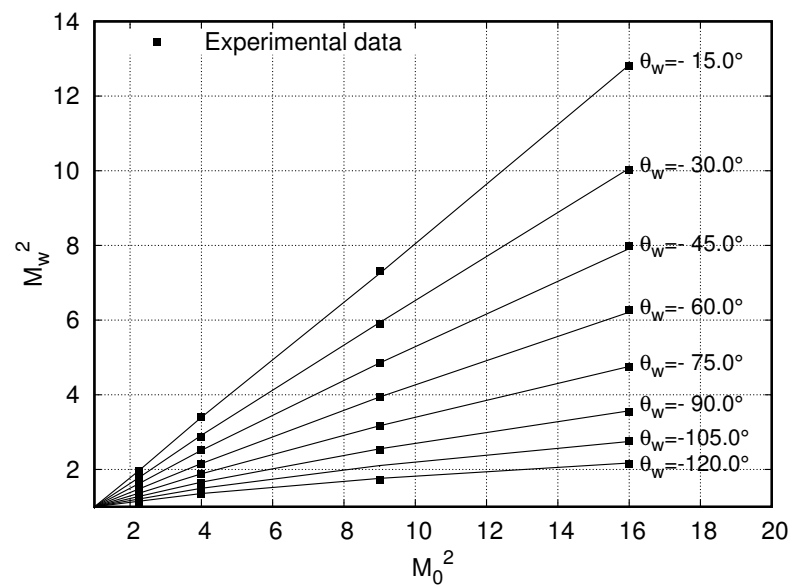

(a)

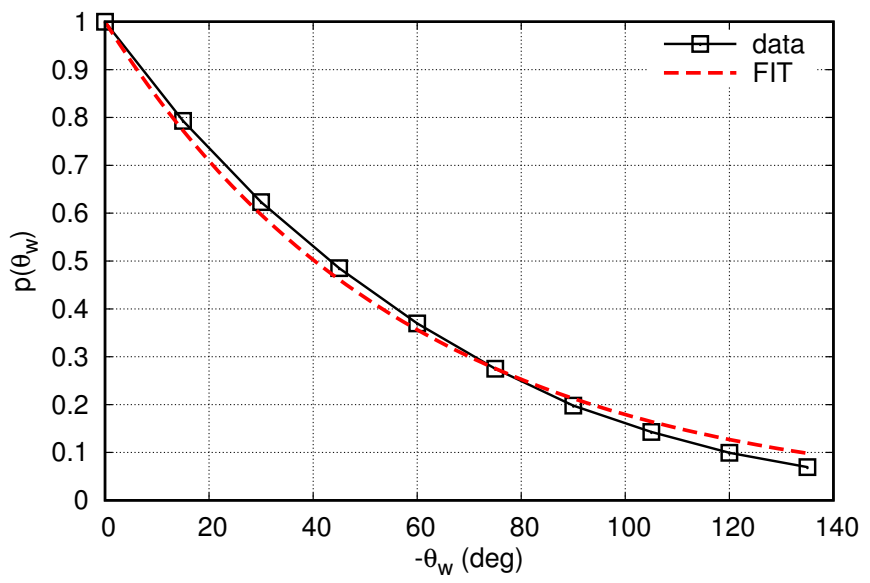

(b)

Figure 7: Fig. (a): $M_{\mathrm{w}}^{2}$ with respect to $M_{0}^{2}$ from Skews' experimental data [29]. Fig. (b): Function $p\left(\theta_{\mathrm{w}}\right)$ from curves $M_{\mathrm{w}}^{2}-1=p\left(\theta_{\mathrm{w}}\right)\left(M_{0}^{2}-1\right)$. Red dashed line is analytical approximation (25).

is self-similar for planar shock diffraction over convex corner, then for a given incident Mach number $M_{0}$ and a pseudo-time $\alpha$, the front shocks share the same rarefaction fan for any deflection angles $\theta_{\mathrm{w}}$ as shown in figure 3. Consequently, we propose that relation (24) holds on each $C^{+}$:

$$
M\left(\theta, M_{0}\right)=\sqrt{1+p(\theta)\left(M_{0}^{2}-1\right)} .
$$

This expression is written under the following differential form between $\theta$ and $M$, available in the rarefaction fan:

$$
\frac{2 M}{M^{2}-1} \frac{\mathrm{d} M}{\mathrm{~d} \alpha}=\frac{p^{\prime}(\theta)}{p(\theta)} \frac{\mathrm{d} \theta}{\mathrm{d} \alpha} \quad \text { on } C^{+}
$$

For the $p$ law (25), the relation (27) yields:

$$
\frac{2 M}{M^{2}-1} \frac{\mathrm{d} M}{\mathrm{~d} \alpha}=k \frac{\mathrm{d} \theta}{\mathrm{d} \alpha} \quad \text { on } C^{+} .
$$

\section{$3.2 \quad$ Ad-hoc modification of the $A-M$ rule}

We propose an ad-hoc modification of Whitham's $A-M$ relation by taking into account the variation of the Mach number along the shock (variation with respect to the curvilinear abscissa, $s$ ). We recall that $s$ and $\beta$ are linked by $\mathrm{d} s=A \mathrm{~d} \beta$. We choose to generalize (4) as:

$$
\frac{1}{A} \frac{\partial A}{\partial \alpha}+\frac{M \lambda(M)}{M^{2}-1} \frac{\partial M}{\partial \alpha}+H(\kappa) f(M)\left|\frac{\partial M}{A \partial \beta}\right|=0
$$

Notice that there is no transverse variation for the radial configuration and consequently (29) reduces to the classical $A-M$ rule in this case. 
Since the GSD model works well in compressive zones [18], the additional term has to be active only in expansive regions of the shock front, where the curvature, $\kappa$, is positive. In this way, the function $H$ is introduced:

$$
H(\kappa)= \begin{cases}0 & \text { if } \kappa \leq 0 \\ 1 & \text { if } \kappa>0\end{cases}
$$

The function $f$ depends only on $M$. It is modeled using the empirical law (28), which leads, after calculus detailed hereafter, to

$$
f(M)=\frac{k \lambda(M)}{2}-\frac{2 M^{2}}{k\left(M^{2}-1\right)}, \text { with } k=0.985 .
$$

The proof of (30) starts by injecting the modified $A-M$ relation (29) in the system (2). One obtains

$$
\left\{\begin{array}{l}
\frac{\partial M}{\partial \alpha}+\frac{M^{2}-1}{\lambda(M) A} \frac{\partial \theta}{\partial \beta}+\eta \frac{f(M)\left(M^{2}-1\right)}{M \lambda(M) A} \frac{\partial M}{\partial \beta}=0 \\
\frac{\partial \theta}{\partial \alpha}+\frac{1}{A} \frac{\partial M}{\partial \beta}=0
\end{array}\right.
$$

where $\eta=H(\kappa) \operatorname{sign}\left(\frac{\partial M}{\partial \beta}\right)$. The eigenvalues of the system $(31), u_{ \pm}$, are given by:

$$
A u_{ \pm}(M)=\frac{\eta_{ \pm} f(M)\left(M^{2}-1\right)}{2 M \lambda(M)} \pm \frac{1}{2} \sqrt{\frac{M^{2}-1}{\lambda(M)}} \sqrt{\frac{\left(\eta_{ \pm}\right)^{2} f(M)^{2}\left(M^{2}-1\right)}{M^{2} \lambda(M)}+4},
$$

with $\eta_{+}$and $\eta_{-}$the values of $\eta$ following respectively the characteristic $C^{+}$and $C^{-}$. The absolute value on the transverse term in (29) ensures the symmetry of the waves: $u_{+}(M)=$ $-u_{-}(M)$. The case of the interaction of two shocks at Mach number $M_{0}$, shown in figure 8, is thus identical to the planar diffraction of a shock over a convex corner presented in figure 2(a). Provided the function $M \mapsto f(M)$ is real, the eigenvalues $u_{ \pm}$are real and the system (31) is hyperbolic. In particular, if $\eta f(M)$ is zero, this system reduces to the GSD model.

The characteristic form of (31) is:

$$
\frac{\mathrm{d} \theta}{\mathrm{d} \alpha} \pm \frac{1}{A u_{+}(M)} \frac{\mathrm{d} M}{\mathrm{~d} \alpha}=0 \quad \text { on } C^{ \pm}: \frac{\mathrm{d} \beta}{\mathrm{d} \alpha}= \pm u_{+}(M)
$$

giving the Riemann invariants:

$$
\theta-\theta_{0} \pm \int_{M_{0}}^{M} \frac{\mathrm{d} m}{A u_{+}(m)}=\operatorname{cst} \quad \text { on } C^{ \pm}
$$

Let us now consider the diffraction of a planar shock over a convex corner as explained in section 2.3. The solution is a simple wave (all $C^{-}$characteristics cross the initial data $\theta=\theta_{0}=0$ and $M=M_{0}$ ) and is completely defined by:

$$
\frac{\mathrm{d} \theta}{\mathrm{d} \alpha}=\frac{1}{A u_{+}(M)} \frac{\mathrm{d} M}{\mathrm{~d} \alpha} \text { on } C^{+} .
$$




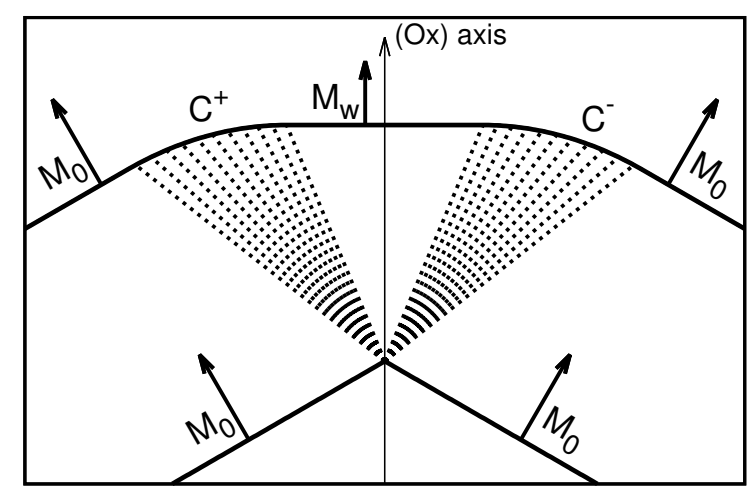

Figure 8: Interaction of two planar shocks at Mach number $M_{0}$ with a direct angle of $\theta_{\mathrm{w}}$ and $-\theta_{\mathrm{w}}$ with respect to the $(O x)$ axis. This problem is identical to the planar shock diffraction over a convex corner sketched in figure $2(\mathrm{a})$.

In order to model the function $f,(32)$ is identified with the empirical law (28):

$$
k \frac{M^{2}-1}{2 M}=A u_{+}(M)=\frac{f(M)\left(M^{2}-1\right)}{2 M \lambda(M)}+\frac{1}{2} \sqrt{\frac{M^{2}-1}{\lambda(M)}} \sqrt{\frac{f(M)^{2}\left(M^{2}-1\right)}{M^{2} \lambda(M)}+4},
$$

as $\eta_{+}=1$ for this problem. After some calculation, the expression (30) follows. Another choice of closure, using a more complicated $p$ law than (25), implies to consider the orientation of the ray, $\theta$, in the $A-M$ relation. Such an expression is not considered here in order to keep a simple expression between $A$ and $M$.

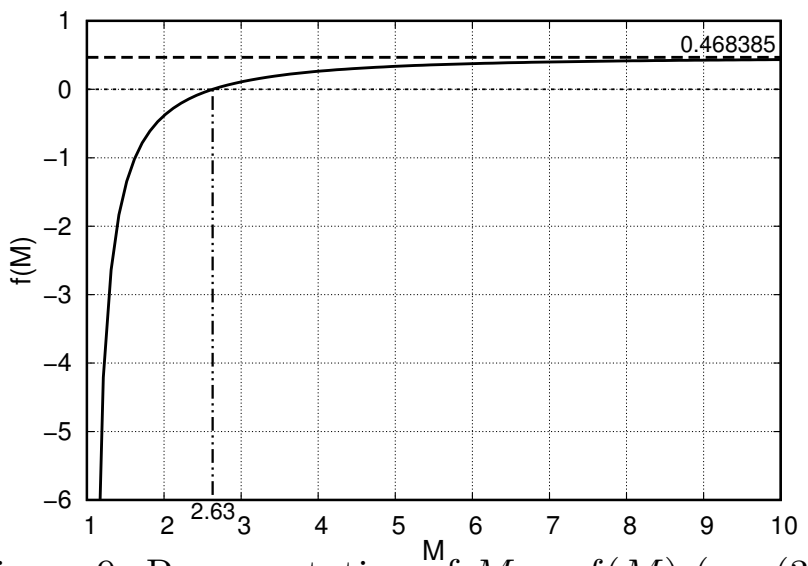

Figure 9: Representation of $M \mapsto f(M)$ (eq. (30)).

The graph of $M \mapsto f(M)$ is drawn in figure 9 . For a strong shock $(M \gg 1), f$ tends to a finite value:

$$
f(M) \sim \frac{k \lambda_{\infty}}{2}-\frac{2}{k} \approx 0.468385
$$


while in the weak shock limit $(M \sim 1), f$ is not bounded:

$$
f(M) \sim-\frac{1}{k(M-1)}
$$

This is a desirable property for overcoming the limitation of the GSD model for weak shocks. For a Mach number lower than $M_{\mathrm{c}} \approx 2.631$, the correction accelerates the shock front in comparison to the original model. Conversely, the shock is slowed down with a Mach number up to $M_{\mathrm{c}}$. The GSD model with the $A-M$ relation (29) will be referred as GSDT model



(a)

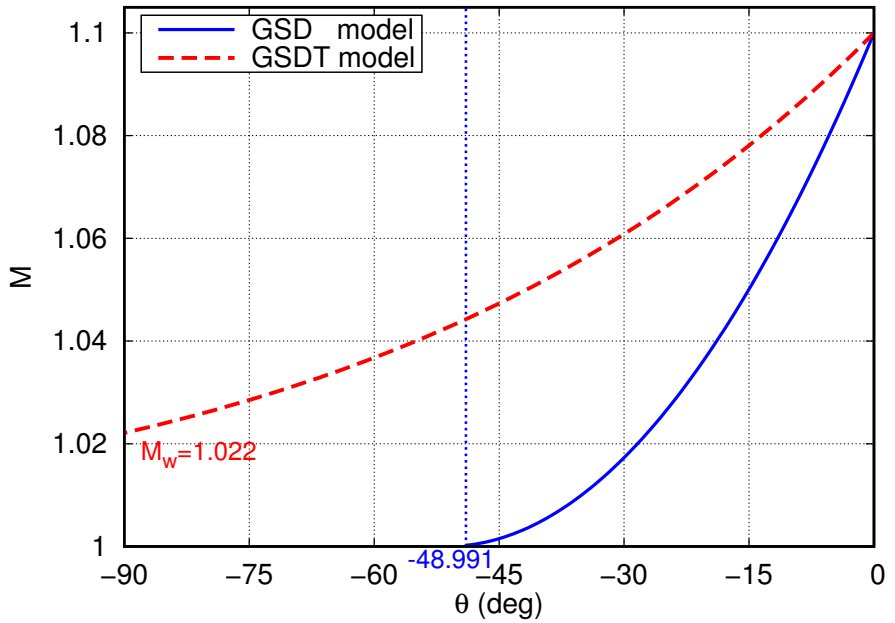

(b)

Figure 10: Diffraction of a planar shock at Mach number $M_{0}=1.1$ over a convex corner of angle $\theta_{\mathrm{w}}=-\pi / 2$. The solution is given by (19)-(20) with (8) for the GSD model and (33) for the GSDT model. Fig. (a): Shock front at $\alpha=1$. Fig. (b): Mach number along the shock.

( $\mathrm{T}$ for Transverse) in the remainder of this article. 


\subsection{A first analytical example}

As a first example, the results for the diffraction of a planar shock at Mach number $M_{0}=1.1$ over a convex corner of angle $-90^{\circ}$ are shown in figure 10. In comparison to figure 5, the solution with GSDT now exists up to the wall. In particular, the Mach number at the wall is around 1.022, calculated with the expression (24)-(25). We can observe the difference of the expansion wave between both models. In this example, the diminution of the Mach number along the shock is smaller with GSDT compared to the GSD model (see Fig. 10(b)). In figure 10(a), we observe a better estimation of the shock front with GSD up to the deflection angle of $-48.991^{\circ}$ compared to an Eulerian simulation (Eulerian simulations characteristics are described in [18]). However, the modified GSDT model does predict a solution with reasonable accuracy beyond this limit angle. The small difference with the Eulerian simulation results is marginal in regard to the gain in terms of new potential applications.

More examples will be presented in section 5.1.

\section{Numerical scheme}

In this section, the numerical scheme aimed at solving the GSDT model for two-dimensional problems is detailed. It is a natural approach for GSD, inspired from Henshaw et al. [7], based on the Lagrangian motion of the shock and on its decomposition in ray tubes. The algorithm dynamically manages the number of points on the front through a process called regularization. Points are inserted in expansive regions to maintain acceptable surface resolution, and are deleted in compressive zones in order to avoid intersecting rays and to fit shock-shocks.

In the original scheme, the shock front is interpolated with a global cubic spline to estimate unit normal vectors at the front. Points are then evolved by integrating the discrete version of (1) with a Leap-Frog scheme initialized with an explicit Euler step. A centered scheme is used to calculate the ray tube area, and a smoothing procedure, called filtering, is applied to dampen the high-frequency errors in the position of points. The Mach number is estimated by integrating the $A-M$ rule in each discrete ray tube from the reference position. This ensures the scheme to be conservative, especially after the regularization step.

We propose some modifications of this Lagrangian scheme in order to improve its robustness and to deal with the transverse term. The global spline interpolation produces oscillations, particularly in regions of large gradient such as the vicinity of a shock-shock. Furthermore, such an interpolation implies a global dependency of data. We substitute this procedure with a local monotone cubic spline [8] which prevents these difficulties. Second, points are propagated with a Total Variation Diminishing (TVD) third order Runge-Kutta scheme [6], which avoids the reinitialization of the scheme after regularization or filtering. Moreover, this method is compatible with a centered scheme and limits oscillations. Last but not least, we adapt the discrete version of the $A-M$ relation in order to integrate it step by step but still keeping the scheme conservative. This allows an easy integration of 
the transverse term (29).

The main steps of the algorithm are described below and are summarized in subsection 4.4.

\subsection{Algorithm description}

Let us consider an initial shock front, at time $\alpha_{0}$, with Cartesian coordinates denoted by:

$$
\boldsymbol{x}\left(\alpha_{0}, \beta\right)=\left(x\left(\alpha_{0}, \beta\right), y\left(\alpha_{0}, \beta\right)\right)^{T} .
$$

The initial Mach number distribution is $M_{0}=M\left(\alpha_{0}, \beta\right)$. It has not to be uniform along the shock. As $\beta$ and the curvilinear abscissa, $s$, are linked by $\mathrm{d} s=A \mathrm{~d} \beta$, we can equivalently consider the curvilinear abscissa.

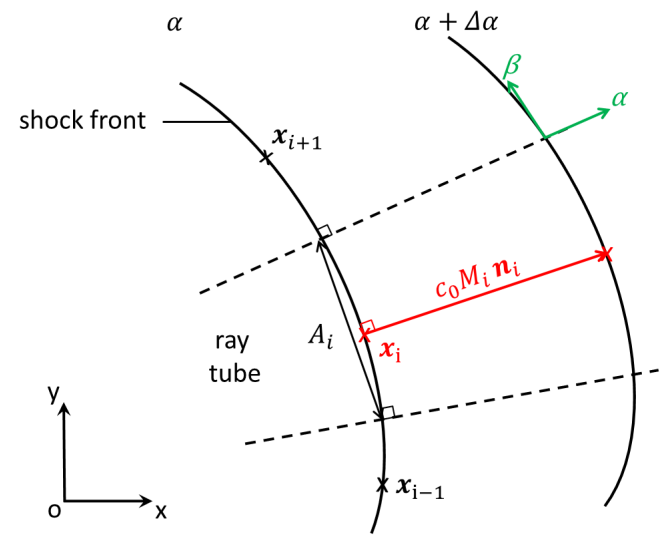

Figure 11: Scheme of the shock front evolution discretization in the Lagrangian scheme.

Let $\left(\beta_{i}\right)_{i=1, \ldots, N}$ be a discretization of the $\beta$ coordinate. The shock front at time $\alpha \geq \alpha_{0}$ is approximated by a finite set of points:

$$
\boldsymbol{x}\left(\alpha, \beta_{i}\right)=\boldsymbol{x}_{i}(\alpha)=\left(x_{i}, y_{i}\right)^{T} \quad \text { for } i=1, \ldots, N .
$$

We introduce the discrete curvilinear abscissa along the shock:

$$
s_{i}= \begin{cases}0 & \text { if } i=1 \\ s_{i-1}+\left\|\vec{x}_{i}-\vec{x}_{i-1}\right\| & \text { if } i=2, \ldots, N,\end{cases}
$$

where $\|\vec{X}\|=\sqrt{x^{2}+y^{2}}$ for $\vec{X}=(x, y)^{T}$, and the space step: $\Delta s_{i}=s_{i}-s_{i-1}$, for $i=2, \ldots, N$. The initial shock discretization is uniform and is defined by:

$$
\Delta s=\frac{1}{N} \sum_{i=2}^{N}\left(s_{i}\left(\alpha_{0}\right)-s_{i-1}\left(\alpha_{0}\right)\right)=\frac{s_{N}\left(\alpha_{0}\right)}{N} .
$$

Each point $\boldsymbol{x}_{i}(\alpha)$ is associated to a ray tube of local area $A_{i}(\alpha)$, a Mach number $M_{i}(\alpha)$, and a unit outward normal vector $\boldsymbol{n}_{i}(\alpha)$. Once all that information are known, points are 
moved to time $\alpha+\Delta \alpha$ by integrating the discrete version of (1), that is

$$
\frac{\mathrm{d} \boldsymbol{x}_{i}}{\mathrm{~d} \alpha}=M_{i} \boldsymbol{n}_{i} \quad \text { for } i=1, \ldots, N
$$

with a TVD third order Runge-Kutta scheme [6]:

$$
\begin{aligned}
\boldsymbol{x}_{i}^{(1)} & =\boldsymbol{x}_{i}(\alpha)+\Delta \alpha M_{i}(\alpha) \boldsymbol{n}_{i}(\alpha) \\
\boldsymbol{x}_{i}^{(2)} & =\frac{3}{4} \boldsymbol{x}_{i}(\alpha)+\frac{1}{4} \boldsymbol{x}_{i}^{(1)}+\frac{\Delta \alpha}{4} M_{i}^{(1)} \boldsymbol{n}_{i}^{(1)} \\
\boldsymbol{x}_{i}(\alpha+\Delta \alpha) & =\frac{1}{3} \boldsymbol{x}_{i}(\alpha)+\frac{2}{3} \boldsymbol{x}_{i}^{(2)}+\frac{2}{3} \Delta \alpha M_{i}^{(2)} \boldsymbol{n}_{i}^{(2)} .
\end{aligned}
$$

The quantities $M_{i}$ and $\boldsymbol{n}_{i}$ are evaluated after each substep using the procedures described hereafter. Figure 11 sketches the discrete shock front propagation.

The time step, $\Delta \alpha$, is restricted by the stability condition of the algorithm, and by the limit on the number of points interacting with a material boundary.

Proposition 1 The scheme is stable under the Courant-Friedrichs-Lewy (CFL) condition:

$$
\Delta \alpha \leq \Delta \alpha_{s}=d_{\min } \Delta s \min _{i=1, \ldots, N}\left(\sqrt{\frac{\lambda\left(M_{i}\right)}{M_{i}^{2}-1}}, \frac{2 M_{i}}{k\left(M_{i}^{2}-1\right)}\right),
$$

where $d_{\min }$ is a parameter introduced after.

Proof 1 This inequality is a consequence of the velocity of the perturbations on the shock front (7):

$$
\frac{\mathrm{d} s}{\mathrm{~d} \alpha}= \pm A u(M)
$$

It is given by (8) for compressive regions and (33) for expansive regions.

Proposition 2 Rays do not cross under the condition:

$$
\Delta \alpha \leq \Delta \alpha_{c}=\min _{i \in \mathcal{I}}\left(\frac{l_{i-1}}{M_{i-1}}, \frac{k_{i}}{M_{i}}\right)
$$

where

$$
\begin{aligned}
l_{i-1} & =\frac{\overrightarrow{\boldsymbol{x}_{i-1} \boldsymbol{x}_{i}} \cdot \vec{n}_{i-1}-\left(\overrightarrow{\boldsymbol{x}_{i-1} \boldsymbol{x}_{i}} \cdot \vec{n}_{i}\right)\left(\vec{n}_{i-1} \cdot \vec{n}_{i}\right)}{1-\left(\vec{n}_{i-1} \cdot \vec{n}_{i}\right)^{2}} \\
k_{i} & =-\frac{\overrightarrow{\boldsymbol{x}_{i-1} \boldsymbol{x}_{i}} \cdot \vec{n}_{i}-\left(\overrightarrow{\boldsymbol{x}_{i-1} \boldsymbol{x}_{i}} \cdot \vec{n}_{i-1}\right)\left(\vec{n}_{i-1} \cdot \vec{n}_{i}\right)}{1-\left(\vec{n}_{i-1} \cdot \vec{n}_{i}\right)^{2}},
\end{aligned}
$$

and

$$
\mathcal{I}=\left\{i=2, \ldots, N / l_{i-1}>0, k_{i}>0 \text { and }\left|\vec{n}_{i-1} \cdot \vec{n}_{i}\right| \neq 1\right\}
$$


Proof 2 Let us consider two neighbouring points on the front, $\boldsymbol{x}_{i-1}$ and $\boldsymbol{x}_{i}$, moving in the direction $\vec{n}_{i-1}$ and $\vec{n}_{i}$ respectively. The possible cross point of the associated lines, $Q$, exists if and only if $\left|\vec{n}_{i-1} \cdot \vec{n}_{i}\right| \neq 1$. We then find $l_{i-1}$ and $k_{i}$ two strictly positive real numbers such that

$$
\overrightarrow{\boldsymbol{x}_{i-1} Q}=l_{i-1} \vec{n}_{i-1} \quad \text { and } \quad \overrightarrow{\boldsymbol{x}_{i} Q}=k_{i} \vec{n}_{i}
$$

We have $\overrightarrow{\boldsymbol{x}_{i-1} \boldsymbol{x}_{i}}=l_{i-1} \vec{n}_{i-1}-k_{i} \vec{n}_{i}$, and by contracting this relation with $\vec{n}_{i-1}$ and $\vec{n}_{i},\left(l_{i-1}, k_{i}\right)$ is solution of the linear system:

$$
\left\{\begin{array}{l}
\overrightarrow{\boldsymbol{x}_{i-1} \boldsymbol{x}_{i}} \cdot \vec{n}_{i-1}=l_{i-1}-k_{i}\left(\vec{n}_{i-1} \cdot \vec{n}_{i}\right) \\
\overrightarrow{\boldsymbol{x}_{i-1} \boldsymbol{x}_{i}} \cdot \vec{n}_{i}=-k_{i}+l_{i-1}\left(\vec{n}_{i-1} \cdot \vec{n}_{i}\right)
\end{array}\right.
$$

This system is invertible provided that $\left|\vec{n}_{i-1} \cdot \vec{n}_{i}\right| \neq 1$, for which (38a)-(38b) is solution. Finally, the inequalities (37) come from a step of Euler integration for $i=2, \ldots, N$.

Finally, the time step is chosen as

$$
\Delta \alpha=\delta \min \left(\Delta \alpha_{s}, \Delta \alpha_{c}\right)
$$

where $\delta \in] 0,1[$ is a safety coefficient chosen equal to 0.5 in practice.

An interpolation of the shock surface is performed over the data $\left(s_{i}, x_{i}\right)_{i=1, \ldots, N},\left(s_{i}, y_{i}\right)_{i=1, \ldots, N}$ using a monotone cubic method [8] in order to avoid the creation of new extrema. The interpolates are denoted $s \mapsto X(s)$ and $s \mapsto Y(s)$ and are $C^{1}$ at least. The unit normal vector is calculated accordingly:

$$
\vec{n}_{i}=\frac{\left(Y^{\prime}\left(s_{i}\right),-X^{\prime}\left(s_{i}\right)\right)^{T}}{\sqrt{X^{\prime}\left(s_{i}\right)^{2}+Y^{\prime}\left(s_{i}\right)^{2}}} \text { for } i=1, \ldots, N
$$

The local area is obtained with a centered scheme for the interior points and a one-sided scheme at the endpoints:

$$
A_{i}=\frac{\pi^{\nu}}{2} \begin{cases}\Delta s_{2}\left(x_{2}+x_{1}\right)^{\nu} & \text { if } i=1 \\ \Delta s_{i+1}\left(x_{i+1}+x_{i}\right)^{\nu} & \\ +\Delta s_{i}\left(x_{i}+x_{i-1}\right)^{\nu} & \text { if } \quad i=2, \ldots, N-1 \\ \Delta s_{N}\left(x_{N}+x_{N-1}\right)^{\nu} & \text { if } \quad i=N,\end{cases}
$$

with $\nu=0$ for a 2D planar configuration and $\nu=1$ for a 2D axisymmetrical flow with $(O y)$ the axis of rotation. The Mach number at time $\alpha+\Delta \alpha$ and at node $\boldsymbol{x}_{i}, M_{i}(\alpha+\Delta \alpha)$, is then obtained by integrating numerically the $A-M$ relation (29) along each ray tube between times $\alpha$ and $\alpha+\Delta \alpha$ :

$$
\log \left(\frac{A_{i}(\alpha+\Delta \alpha)}{A_{i}(\alpha)}\right)+\int_{M_{i}(\alpha)}^{M_{i}(\alpha+\Delta \alpha)} \frac{m \lambda(m)}{m^{2}-1} \mathrm{~d} m+\int_{\alpha}^{\alpha+\Delta \alpha} H(\kappa) f(M)\left|\frac{\partial M}{\partial s}\right| \mathrm{d} \tau=0 .
$$


The resolution of (43) will be presented in subsection 4.2.

After the step of propagation to time $\alpha+\Delta \alpha$, points are inserted in expansive regions to maintain acceptable surface resolution, and are deleted in compressive regions in order to avoid the excessive accumulation of points and fit shock-shocks. For $i=2, \ldots, N$, we require that the point spacing verifies:

$$
d_{\min } \Delta s \leq \Delta s_{i} \leq d_{\max } \Delta s
$$

where $d_{\min }=\frac{1}{2}$ and $d_{\max }=\frac{3}{2}$ as in the original scheme. If $\Delta s_{i}<d_{\min } \Delta s$, the point $\boldsymbol{x}_{i}$ is removed. If $\Delta s_{i}>d_{\max } \Delta s$, a new point, $\boldsymbol{x}_{i-\frac{1}{2}}$, is added using the cubic spline interpolation evaluated at $\frac{1}{2}\left(s_{i-1}+s_{i}\right)$. In such cases, the same points are added or removed on the initial shock front in order to preserve the area ratio $\frac{A_{i}(\alpha+\Delta \alpha)}{A_{i}\left(\alpha_{0}\right)}$. This step implies to rewrite (43) to ensure the conservativity of the scheme and will be detailed in subsection 4.2.

Finally, a simple smoothing procedure is applied to dampen the high-frequency errors in $\boldsymbol{x}_{i}$ as proposed by Henshaw et al. [7]. After $n_{s}$ iterations (between 10 and 50 in practice), we let for $i=2, \ldots, N-1$ :

$$
\boldsymbol{x}_{i} \leftarrow \frac{h_{r}}{h_{l}+h_{r}} \boldsymbol{x}_{i-1}+\frac{h_{l}}{h_{l}+h_{r}} \boldsymbol{x}_{i+1}
$$

with

$$
h_{l}=\left\|\vec{x}_{i}-\vec{x}_{i-1}\right\| \quad \text { and } \quad h_{r}=\left\|\vec{x}_{i+1}-\vec{x}_{i}\right\|,
$$

where we start with $i$-even, and then $i$-odd. The smoothing procedure (45) is a first order approximation of the discrete system of equations:

$$
\frac{\mathrm{d}^{2} \boldsymbol{x}_{i}}{\mathrm{~d} s^{2}} \equiv 0 \quad \text { for } i=1, \ldots, N
$$

for a non-uniform distribution of points, and a second order approximation otherwise.

In the presence of obstacles, wall boundary conditions must be applied to keep the shock locally normal to the wall. This is achieved by clamping the spline interpolation at the boundary:

$$
X^{\prime}\left(s_{\text {bound }}\right)=N_{x} \quad \text { and } \quad Y^{\prime}\left(s_{\text {bound }}\right)=N_{y},
$$

where $s_{\text {bound }}$ defines the shock boundary and $\boldsymbol{N}=\left(N_{x}, N_{y}\right)^{T}$ is the unit normal vector to the wall. In our implementation, the shock can not split and so $s_{\text {bound }}=0$ or $s_{\text {bound }}=s_{N}$. The normal vector at $s_{\text {bound }}$ is then defined by:

$$
\boldsymbol{n}\left(s_{\text {bound }}\right)=\left(N_{y},-N_{x}\right)^{T},
$$

which forces the point $\boldsymbol{x}\left(s_{\text {bound }}\right)$ to move along the wall. For free boundary at the edge of the shock, the spline is natural:

$$
X^{\prime \prime}\left(s_{\text {bound }}\right)=0 \quad \text { and } \quad Y^{\prime \prime}\left(s_{\text {bound }}\right)=0
$$




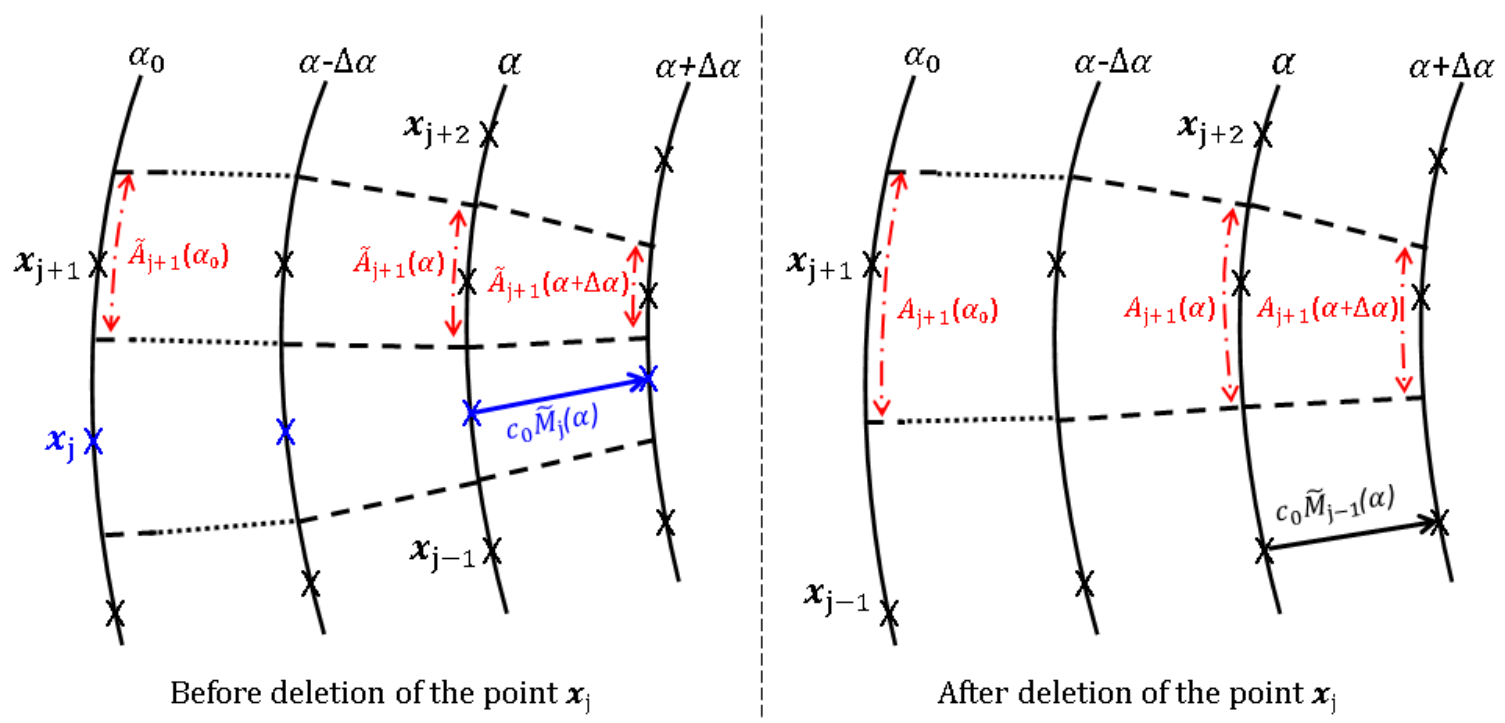

Figure 12: Redefinition of the ray tubes after deletion of the point $\boldsymbol{x}_{j}$.

\subsection{Integration of the $A-M$ relation}

The addition or deletion of some points on the shock front can lead to a loss of conservativity of the scheme. Let us explain this point for the GSD model, that is with $H \equiv 0$ in (29).

In the original algorithm [7], this difficulty is avoided by integrating the $A-M$ relation (4) from the initial time $\alpha_{0}$ to $\alpha+\Delta \alpha$ :

$$
\log \left(\frac{A_{i}(\alpha+\Delta \alpha)}{A_{i}\left(\alpha_{0}\right)}\right)+\int_{M_{i}\left(\alpha_{0}\right)}^{M_{i}(\alpha+\Delta \alpha)} \frac{m \lambda(m)}{m^{2}-1} \mathrm{~d} m=0
$$

as all the information is known at $\alpha_{0}$. As long as there is no regularization, (46) is similar to the integration of (4) from $\alpha_{0}$ to $\alpha$, followed by the integration from time $\alpha$ to $\alpha+\Delta \alpha$. Knowing the information at time $\alpha$, the Mach number at time $\alpha+\Delta \alpha$ can be calculated with

$$
\log \left(\frac{A_{i}(\alpha+\Delta \alpha)}{A_{i}(\alpha)}\right)+\int_{M_{i}(\alpha)}^{M_{i}(\alpha+\Delta \alpha)} \frac{m \lambda(m)}{m^{2}-1} \mathrm{~d} m=0 .
$$

However, this is no longer the case after regularization.

Let us assume that the front is regularized for the first time at $\alpha+\Delta \alpha$, and suppose that the point $\boldsymbol{x}_{j}$ is removed. The ray tubes indexed by $j-1$ and $j+1$ are then modified as sketched in figure 12 , and consequently $A_{j-1}$ and $A_{j+1}$ have changed. Let us denote $\tilde{\psi}$ a quantity before regularization, and $\psi$ this quantity after. Both quantities are equal for the initial data: $\psi\left(\alpha_{0}\right)=\tilde{\psi}\left(\alpha_{0}\right)$. At node $\boldsymbol{x}_{j+1}$, the Mach number at $\alpha+\Delta \alpha$ should be evaluated with

$$
\log \left(\frac{A_{j+1}(\alpha+\Delta \alpha)}{A_{j+1}(\alpha)}\right)+\int_{\tilde{M}_{j+1}(\alpha)}^{M_{j+1}(\alpha+\Delta \alpha)} \frac{m \lambda(m)}{m^{2}-1} \mathrm{~d} m=0
$$


using (47). Its value must be the same with an integration from the initial time (46):

$$
\log \left(\frac{A_{j+1}(\alpha+\Delta \alpha)}{A_{j+1}\left(\alpha_{0}\right)}\right)+\int_{M_{j+1}\left(\alpha_{0}\right)}^{M_{j+1}(\alpha+\Delta \alpha)} \frac{m \lambda(m)}{m^{2}-1} \mathrm{~d} m=0
$$

The Mach number at time $\alpha$ is known before regularization and can be calculated with:

$$
\log \left(\frac{\tilde{A}_{j+1}(\alpha)}{\tilde{A}_{j+1}\left(\alpha_{0}\right)}\right)+\int_{M_{j+1}\left(\alpha_{0}\right)}^{\tilde{M}_{j+1}(\alpha)} \frac{m \lambda(m)}{m^{2}-1} \mathrm{~d} m=0 .
$$

Applying (50) and then (48) yields:

$$
\log \left(\frac{A_{j+1}(\alpha+\Delta \alpha)}{A_{j+1}\left(\alpha_{0}\right)}\right)+\int_{M_{j+1}\left(\alpha_{0}\right)}^{M_{j+1}(\alpha+\Delta \alpha)} \frac{m \lambda(m)}{m^{2}-1} \mathrm{~d} m+\log \left(\frac{\tilde{A}_{j+1}(\alpha)}{A_{j+1}(\alpha)} \frac{A_{j+1}\left(\alpha_{0}\right)}{\tilde{A}_{j+1}\left(\alpha_{0}\right)}\right)=0
$$

which differs from (49) due to the modification of local areas. We have thus a loss of information which leads to a loss of conservativity of the scheme. The reasoning is similar for inserted points. Therefore, the integration of the $A-M$ relation from $\alpha$ to $\alpha+\Delta \alpha$ needs a modification.

The following properties ensure the conservativity of the scheme for an $A-M$ relation with the general form:

$$
\frac{1}{A} \frac{\partial A}{\partial \alpha}+\frac{M \lambda(M)}{M^{2}-1} \frac{\partial M}{\partial \alpha}+R(\alpha, \beta)=0 .
$$

The relation (29) is found with

$$
R(\alpha, \beta)=H(\kappa) f(M)\left|\frac{\partial M}{\partial s}\right| .
$$

Proposition 3 (For the deleted points) Let $\tilde{A}_{i}$ and $\tilde{M}_{i}$ be the area and Mach number associated to the point $\boldsymbol{x}_{i}$ before regularization, and let $A_{i}$ and $M_{i}$ be these same quantities after. For each point except the inserted points, the information in ray tubes is conserved with the relation:

$$
\log \left(\frac{A_{i}(\alpha+\Delta \alpha)}{\tilde{A}_{i}(\alpha)} \frac{\tilde{A}_{i}\left(\alpha_{0}\right)}{A_{i}\left(\alpha_{0}\right)}\right)+\int_{\tilde{M}_{i}(\alpha)}^{M_{i}(\alpha+\Delta \alpha)} \frac{m \lambda(m)}{m^{2}-1} \mathrm{~d} m+\int_{\alpha}^{\alpha+\Delta \alpha} R\left(\tau, \beta_{i}\right) \mathrm{d} \tau=0 .
$$

Proof 3 The conservativity of the scheme is ensured integrating the $A-M$ relation (51) from initial time:

$$
\log \left(\frac{A_{i}(\alpha+\Delta \alpha)}{A_{i}\left(\alpha_{0}\right)}\right)+\int_{M_{i}\left(\alpha_{0}\right)}^{M_{i}(\alpha+\Delta \alpha)} \frac{m \lambda(m)}{m^{2}-1} \mathrm{~d} m+\int_{\alpha_{0}}^{\alpha+\Delta \alpha} R\left(\tau, \beta_{i}\right) \mathrm{d} \tau=0 .
$$


The Mach number at time $\alpha$ is known before regularization:

$$
\log \left(\frac{\tilde{A}_{i}(\alpha)}{\tilde{A}_{i}\left(\alpha_{0}\right)}\right)+\int_{M_{i}\left(\alpha_{0}\right)}^{\tilde{M}_{i}(\alpha)} \frac{m \lambda(m)}{m^{2}-1} \mathrm{~d} m+\int_{\alpha_{0}}^{\alpha} R\left(\tau, \beta_{i}\right) \mathrm{d} \tau=0 .
$$

Relation (52) comes from subtracting (54) to (53).

For an inserted point $\boldsymbol{x}_{i+1 / 2}$, the relation (52) can not be used as $M_{i+1 / 2}(\alpha)$ is unknown. The information at points $\boldsymbol{x}_{i}$ and $\boldsymbol{x}_{i+1}$ is then considered.

Proposition 4 (For the inserted points) Let us denote $A_{i}$ and $M_{i}$ the area and Mach number associated to the point $\boldsymbol{x}_{i}$ after regularization. If $\boldsymbol{x}_{i+1 / 2}$ is inserted on the shock front at time $\alpha+\Delta \alpha$, the Mach number $M_{i+1 / 2}(\alpha+\Delta \alpha)$ can be estimated at first order of truncation with the following relation:

$$
I+\int_{M_{i}(\alpha+\Delta \alpha)}^{M_{i+1 / 2}(\alpha+\Delta \alpha)} \frac{m \lambda(m)}{m^{2}-1} \mathrm{~d} m+\int_{M_{i+1}(\alpha+\Delta \alpha)}^{M_{i+1 / 2}(\alpha+\Delta \alpha)} \frac{m \lambda(m)}{m^{2}-1} \mathrm{~d} m=0,
$$

where

$$
\begin{aligned}
I=2 \log \left(\frac{A_{i+1 / 2}(\alpha+\Delta \alpha)}{A_{i+1 / 2}\left(\alpha_{0}\right)} \sqrt{\frac{A_{i}\left(\alpha_{0}\right)}{A_{i}(\alpha+\Delta \alpha)} \frac{A_{i+1}\left(\alpha_{0}\right)}{A_{i+1}(\alpha+\Delta \alpha)}}\right) & \\
& +\int_{M_{i+1 / 2}\left(\alpha_{0}\right)}^{M_{i}\left(\alpha_{0}\right)} \frac{m \lambda(m)}{m^{2}-1} \mathrm{~d} m+\int_{M_{i+1 / 2}\left(\alpha_{0}\right)}^{M_{i+1}\left(\alpha_{0}\right)} \frac{m \lambda(m)}{m^{2}-1} \mathrm{~d} m .
\end{aligned}
$$

Proof 4 At time $\alpha+\Delta \alpha$, the Mach number at nodes $\boldsymbol{x}_{i}, \boldsymbol{x}_{i+1 / 2}$ and $\boldsymbol{x}_{i+1}$ verifies exactly the relation, denoted $\mathcal{R}_{k}$ :

$$
\log \left(\frac{A_{k}(\alpha+\Delta \alpha)}{A_{k}\left(\alpha_{0}\right)}\right)+\int_{M_{k}\left(\alpha_{0}\right)}^{M_{k}(\alpha+\Delta \alpha)} \frac{m \lambda(m)}{m^{2}-1} \mathrm{~d} m+\int_{\alpha_{0}}^{\alpha+\Delta \alpha} R\left(\tau, \beta_{k}\right) \mathrm{d} \tau=0
$$

for $k=i, k=i+1 / 2$ and $k=i+1$. The weighted sum $\mathcal{R}_{i+1 / 2}-\frac{1}{2} \mathcal{R}_{i}-\frac{1}{2} \mathcal{R}_{i+1}$ gives (55) with the supplementary term:

$$
\int_{\alpha_{0}}^{\alpha+\Delta \alpha}\left\{R\left(\tau, \beta_{i+1 / 2}\right)-\frac{R\left(\tau, \beta_{i}\right)+R\left(\tau, \beta_{i+1}\right)}{2}\right\} \mathrm{d} \tau .
$$

As $R\left(\tau, \beta_{i+1 / 2}\right)=\frac{R\left(\tau, \beta_{i}\right)+R\left(\tau, \beta_{i+1}\right)}{2}+O\left(\Delta \beta^{2}\right)$, this term can be neglected at order 1 . 
Finally, the Mach number at time $\alpha+\Delta \alpha$ is estimated with the following procedure which ensures that the scheme is conservative:

1. First, (52) is applied to all points except newly added points.

2. Secondly, we use (55) for these inserted points.

This procedure is similar to the integration from the initial time. If no regularization has been made, we find the original integration:

$$
\log \left(\frac{A_{i}(\alpha+\Delta \alpha)}{A_{i}(\alpha)}\right)+\int_{M_{i}(\alpha)}^{M_{i}(\alpha+\Delta \alpha)} \frac{m \lambda(m)}{m^{2}-1} \mathrm{~d} m+\int_{\alpha}^{\alpha+\Delta \alpha} R\left(\tau, \beta_{i}\right) \mathrm{d} \tau=0, \quad \text { for } i=1, \ldots, N
$$

This section then discusses the numerical integration of (43). The transverse term is rewritten as:

$$
f(M)\left|\frac{\partial M}{A \partial \beta}\right|=\operatorname{sign}(f(M))\left|\frac{\partial F(M)}{\partial s}\right|,
$$

where $\operatorname{sign}(x)=1$ if $x>0$ and -1 otherwise, and with $F(M)=\int f(m) \mathrm{d} m$. This one is approximated at first order with a monotone upwind scheme inspired from the numerical Godunov Hamiltonian [13]:

$$
f(M)\left|\frac{\partial M}{\partial s}\right|_{\mid M=M_{i}} \approx \operatorname{sign}\left(f\left(M_{i}\right)\right) \max \left(\Delta_{l} F_{i},-\Delta_{r} F_{i}, 0\right)
$$

where $\Delta_{l} F_{i}$ and $\Delta_{r} F_{i}$ are approximate slopes of $F(M)$ at left and right from $M_{i}$ :

$$
\Delta_{l} F_{i}=\frac{F\left(M_{i}\right)-F\left(M_{i-1}\right)}{s_{i}-s_{i-1}}, \Delta_{r} F_{i}=\frac{F\left(M_{i+1}\right)-F\left(M_{i}\right)}{s_{i+1}-s_{i}} .
$$

For the interaction with an obstacle, a rigid wall condition is applied at the shock boundary. We suppose that the shock is locally plane at the edges of the front. Consequently, we impose that the transverse variation of the Mach number at a shock boundary is equal to zero:

$$
\left.\frac{\partial F(M)}{\partial s}\right|_{s=s_{\text {bound }}}=0
$$

On each ray tube, the integration of the $A-M$ relation (29) from time $\alpha$ to $\alpha+\Delta \alpha$ is written as:

$$
\log \left(\frac{A_{i}(\alpha+\Delta \alpha)}{A_{i}(\alpha)}\right)+\int_{M_{i}(\alpha)}^{M_{i}(\alpha+\Delta \alpha)} \frac{m \lambda(m)}{m^{2}-1} \mathrm{~d} m+\int_{\alpha}^{\alpha+\Delta \alpha} \eta_{i} \max \left(\Delta_{l} F_{i},-\Delta_{r} F_{i}, 0\right) \mathrm{d} \tau=0
$$

with $\eta_{i}=H\left(\kappa_{i}\right) \operatorname{sign}\left(f\left(M_{i}\right)\right)$. In fact, this integration should be rewritten in order to preserve areas as explained in properties 3 and 4 . The first integral is calculated with a 
Simpson's rule and the second one with a trapezoidal rule. Finally, knowing $\log \left(\frac{A_{i}(\alpha+\Delta \alpha)}{A_{i}(\alpha)}\right)$, $\kappa_{i}$ and $M_{i}(\alpha)$, for $i=1, \ldots, N$, the vector $\left(M_{i}(\alpha+\Delta \alpha)\right)_{i=1, \ldots, N}$ is solution of a nonlinear system which is solved with a Newton algorithm. The estimation of the local curvature of the front is detailed in subsection 4.3. Let us remark that without the transverse flow in the $A-M$ relation (29), equations in (59) are independent from one other, and we just need to solve $N$ nonlinear equations.

\subsection{Estimation of the local curvature of the shock front}

The resolution of (59) requires the knowledge of the sign of the shock local curvature $\kappa_{i}$ at point $\boldsymbol{x}_{i}$. It can be estimated for $2 \mathrm{D}$ planar configuration through geometrical consideration [19]:

$$
\kappa_{i}=\frac{4 \sin \left(\varphi_{i} / 2\right)}{\left\|\overrightarrow{\boldsymbol{x}_{i-1} \boldsymbol{x}_{i}}\right\|+\left\|\overrightarrow{\boldsymbol{x}_{i} \boldsymbol{x}_{i+1}}\right\|},
$$

where $\varphi_{i}$ is the direct angle between $\overrightarrow{\boldsymbol{x}_{i-1} \boldsymbol{x}_{i}}$ and $\overrightarrow{\boldsymbol{x}_{i} \boldsymbol{x}_{i+1}}$. The discrete local curvature (60) is zero if points $\boldsymbol{x}_{i-1}, \boldsymbol{x}_{i}$ and $\boldsymbol{x}_{i+1}$ are aligned, positive if the points arrangement is convex (expansive area of the shock) and negative otherwise (compressive area of the shock), as illustrated in figures 13. As only the sign of the local curvature is necessary, expression (60)

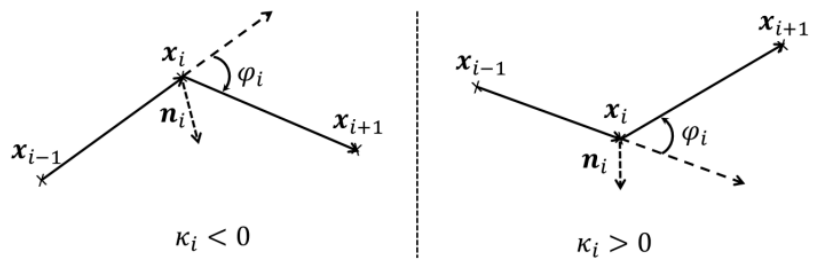

Figure 13: Concave (figure at left) and convexe (figure at right) points arrangement.

can be used for 2D axisymmetrical flow as well.

Formula (60) is sensitive to the small local oscillations of the scheme. Therefore, the sign of $\kappa_{i}$ can be locally negative in expansive regions of the front, and positive for compressive areas, whereas a global inverse behaviour is expected.

To overcome this weakness, we define a temporary coarse distribution of points on the shock front in order to evaluate the curvature. For this purpose, we use a line simplification like algorithm [28], and the intermediate points are interpolated with a monotone quadratic spline [10]. This method has the advantage to preserve the local sign of the curvature, contrary to a cubic spline for example. The local curvature at $\boldsymbol{x}_{i}$ is then estimated using (60) at temporary points.

In the vicinity of a discontinuity like an inflection point (see Fig. 14), the activation of the transverse term can lead to a non-convergence of the numerical solution. The Mach number variation along the shock is indeed very large in such a region. Consequently, we found necessary to deactivate the transverse closure in this area. In such a region, we identify the point where the Mach number variation is the highest, noted $\boldsymbol{X}_{c}$. The transverse term at node $\boldsymbol{x}_{l}$ at a distance $d_{\mathrm{c}}$ from $\boldsymbol{X}_{c}$ are then deactivated: $H\left(\kappa_{l}\right)=0$. The parameter $d_{\mathrm{c}}$ 


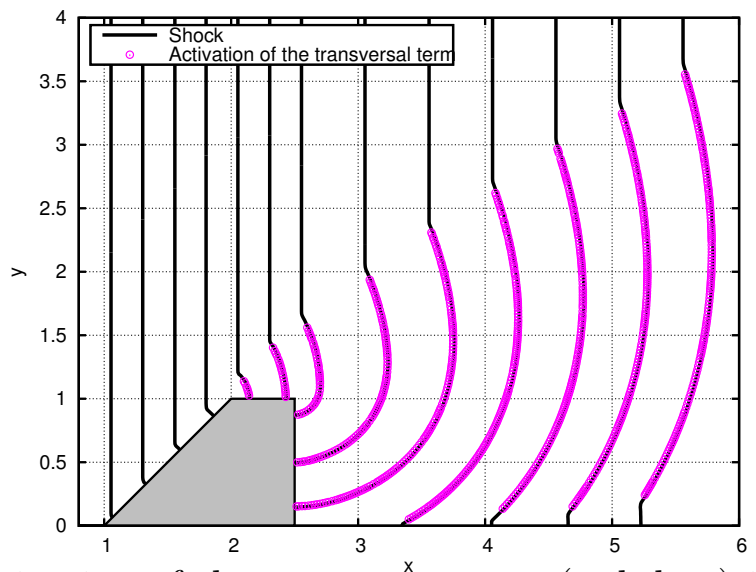

Figure 14: Zones of activation of the transverse term (red dots) in expansive regions of the shock front for the planar shock diffraction over a mound at Mach number $2, \Delta s=0.02$, $d_{\mathrm{c}}=0.05$.

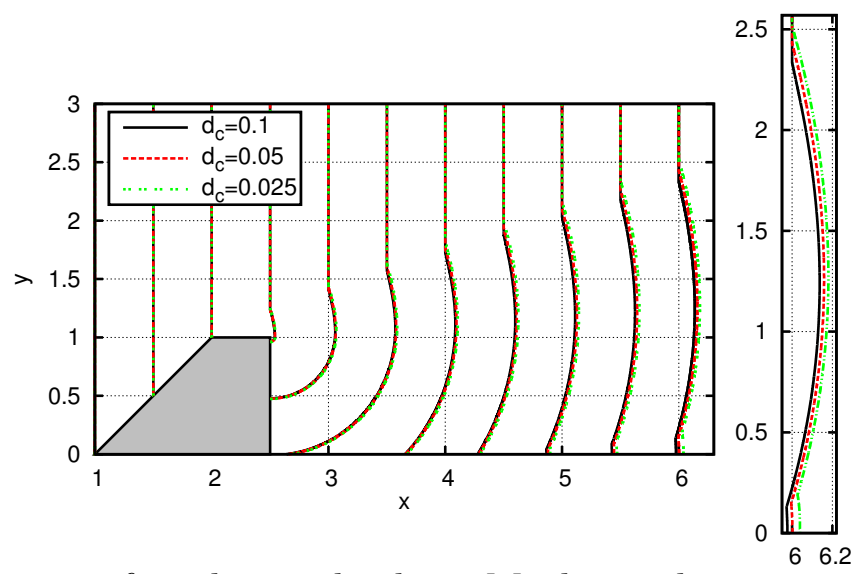

Figure 15: Diffraction of a planar shock at Mach number 1.05 over a mound. Numerical solution for $\Delta s=0.00125$ and for different values of parameter $d_{\mathrm{c}}$.

must be chosen carefully: large enough to ensure numerical convergence of the solution but small enough to still activate the transverse term. In practice, the numerical solution slightly depends on this parameter as can be seen in figure 15. This parameter is taken equal to 0.05 for the examples considered in this paper.

Figure 14 materializes the regions on the shock front where the closure is active for the diffraction of a planar shock over a mound at Mach number 2.

\subsection{Summary of the algorithm}

The numerical scheme we designed to solve the GSDT model from time $\alpha_{0}$ to $\alpha_{\text {end }}$ is now summarized, see Algorithm 1. The algorithm can also be applied to GSD by setting $H \equiv 0$. 


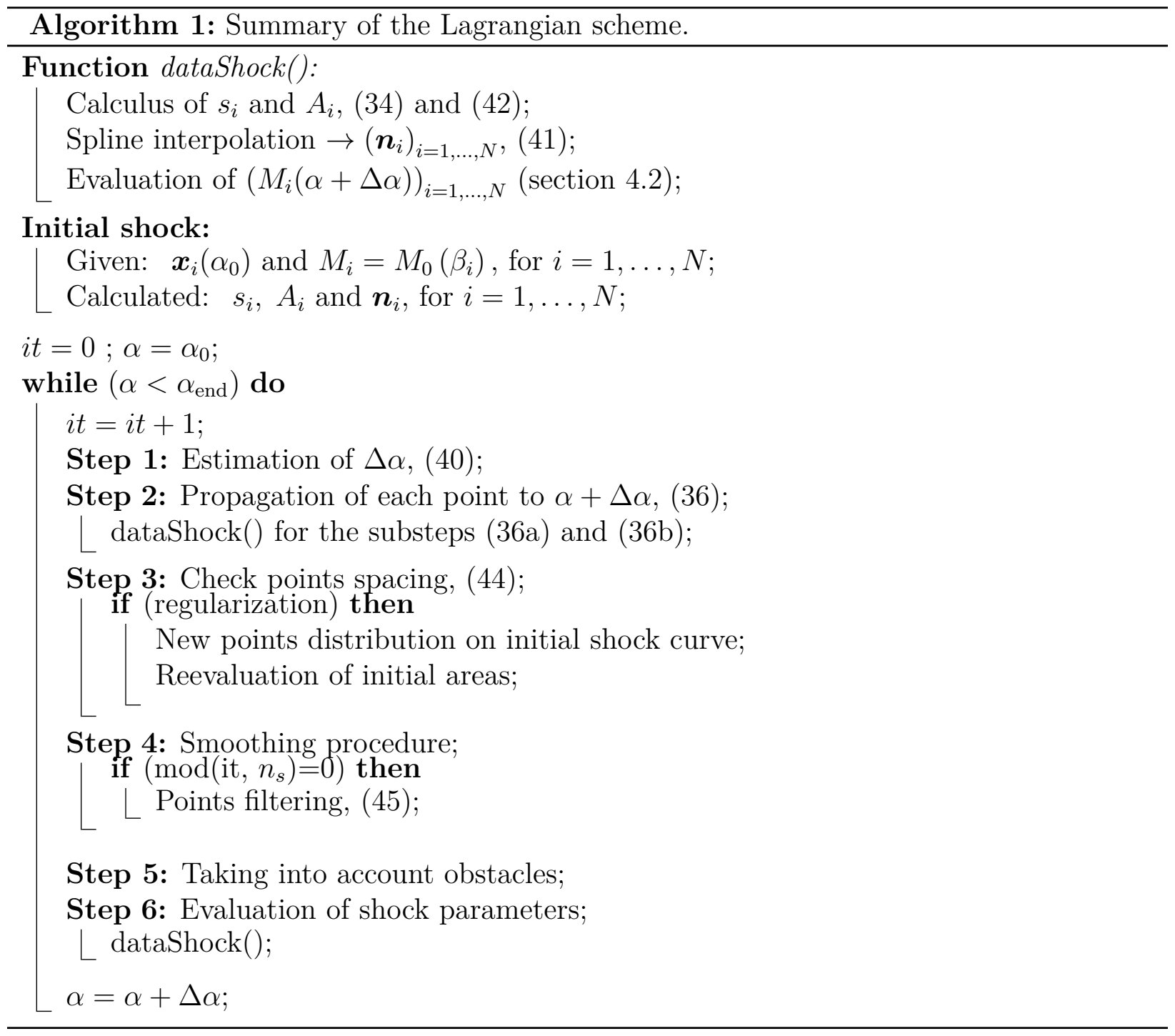

\subsection{Validation of the algorithm for GSDT}

The Lagrangian algorithm is now validated on a set of test cases of increasing complexity. For each of them, a reference solution is known in sections 2.2 and 2.3. For the elementary expansive case, the velocity of the perturbations, $A u$, is defined by (33) for GSDT.

The mesh convergence is first observed for a cylindrical expanding shock. As underlined previously, GSDT is reduced to GSD in this case. The numerical solutions are obtained with the full $A-M$ relation (29). Then, we present an example of a planar shock diffraction over a convex corner and a concave corner. In this last elementary case, we recall that solutions for GSDT are identical to GSD ones. We finish this section with the numerical verification of the conservativity of the scheme. 


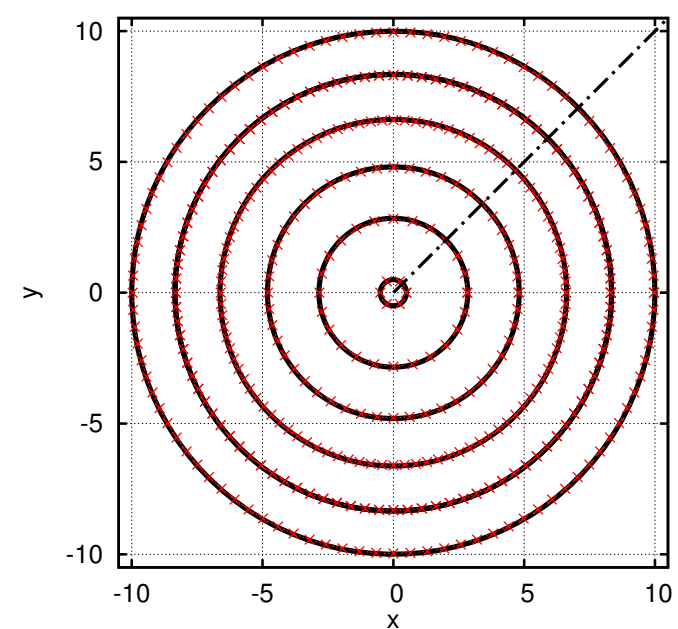

(a)

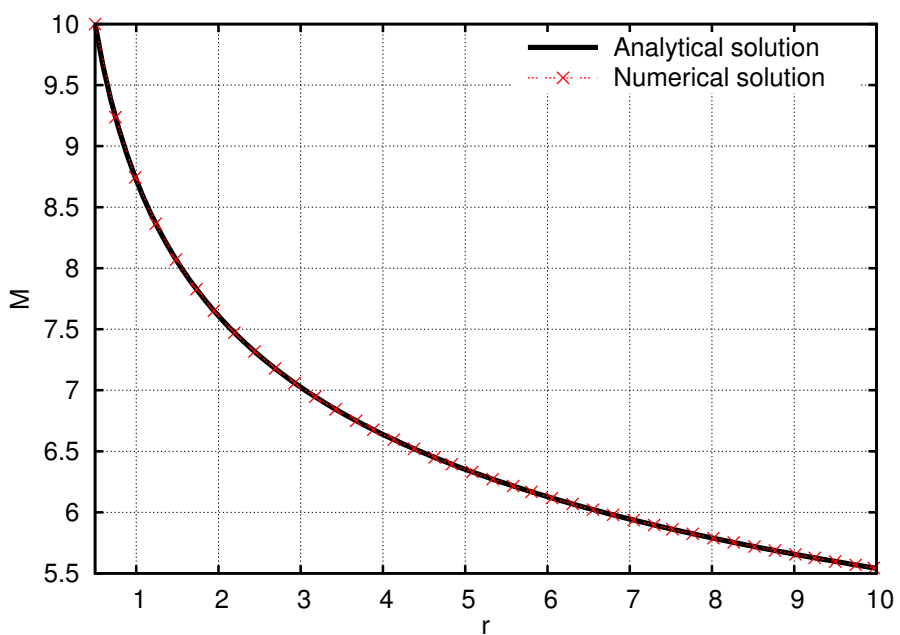

(b)

Figure 16: Successive positions of a cylindrical expanding strong shock $(d=2$, Fig. (a)) and Mach number along the direction of direct angle $\pi / 4$ (Fig. (b)). Comparison between the analytical solution (15) and the numerical results for $\Delta s=0.1$. Initial data: $r_{0}=0.5$ and $M_{0}=10$.

Order of the scheme. We consider the cylindrical expanding shock $(d=2)$. The analytical solution is available in the strong shock limit (15). At initial time $\alpha_{0}=0$, the radius is $r_{0}=0.5$ and the Mach number is $M_{0}=10$. The Lagrangian algorithm is stopped when the shock reaches $r=10$, namely $\alpha_{\text {end }} \approx 0.147$. The successive shock positions and the Mach number decreasing in the radial direction are shown in figure 16. We can observe the periodical insertion of points on the shock front in figure 16(a). The distance to the center of symmetry, $r$, and the Mach number, $M$, are extracted from numerical data at different times $\alpha$ and constant direction of direct angle $\pi / 4$. Figure 17 presents the error of the scheme in the $l^{2}$ norm for several discretization steps, $\Delta s$. As expected, we denote that the order of convergence is 1 owing to the smoothing procedure and the approximation of the transverse term (58). We can notice that the error decreases with the filtering frequency, $f_{s}$, but the convergencce of the scheme is no more monotonous. Results are similar for a spherical shock $(d=3)$ in a $2 \mathrm{D}$-axisymmetrical configuration. In the sequel, the shock front is filtered every 10 iterations.

Planar shock diffraction over a convex wedge. Let us consider the diffraction of a planar shock at Mach number $M_{0}=3$ over a convex corner of deflection angle $\theta_{\mathrm{w}}=$ $-\pi / 2$. The abrupt variation of geometry renders the numerical approximation of the solution difficult near the wall. Nevertheless, one can notice the good agreement of the numerical solution, obtained for $\Delta s=0.0025$, with the analytical solution (19)-(20) in figure 18.

Conservativity of the scheme. To finish, let us study the diffraction of a planar shock at Mach number $M_{0}$ over a concave corner of deflection angle $\theta_{\mathrm{w}}>0$. We recall that the 


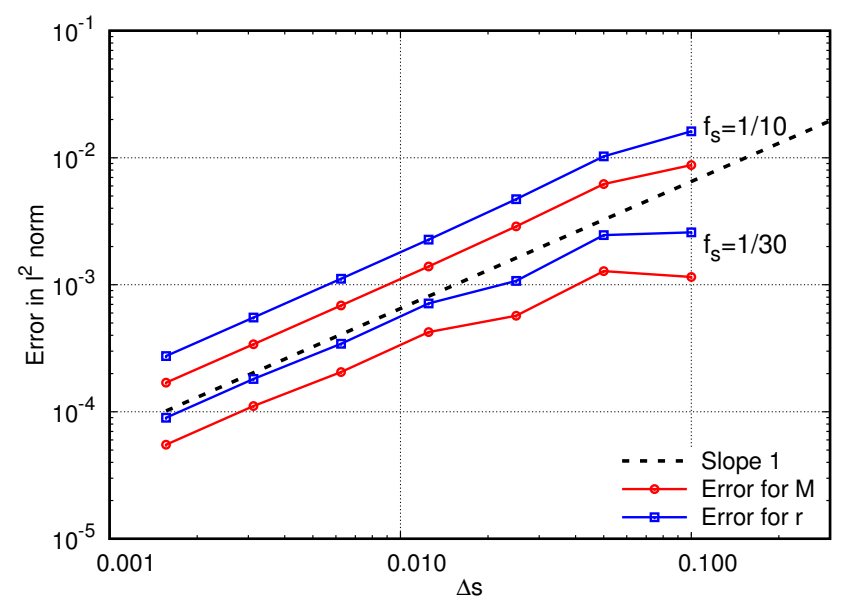

Figure 17: Mesh convergence in the $l^{2}$ norm for the cylindrical expanding strong shock solution with filtering every 10 and 30 iterations.

problem is sketched in figure 2(b) and the solution is given by (21). An example for $M_{0}=3$ and $\theta_{\mathrm{w}}=\pi / 4$ is presented in figure 19. We can see the good restitution of the solution in spite of some oscillations of the scheme observed in figure 19(b) near the shock-shock. In figure 20, one can observe the excellent agreement between results given by the scheme and the analytical solution (21). The wall Mach numbers and shock-shock trajectories are compared for a large range of deflection angles, and for a strong shock $\left(M_{0}=10\right)$ as well as a weaker one $\left(M_{0}=1.5\right)$. The Lagrangian simulations are computed with $\Delta s=0.01$. This discretization step is small enough to estimate correctly the shock-shock velocity. Consequently, the method catches the discontinuity of the model which proves the conservativity of the GSDT algorithm.

\section{Results}

In this section, we present some comparisons between GSD and GSDT models. We start with the elementary problem of the planar diffraction over a convex wall, for which analytical solutions are available. The analysis follows with the diffraction of a planar shock over a turned mound in the second subsection, and over a mound in the last subsection. For these last cases, the Lagrangian scheme presented in section 4 is used to solve GSD and GSDT. The results are compared with experimental data or Eulerian simulations. The latter are provided by the HERA Computational Fluid Dynamics software [9, 18]. During a simulation, the first arrival time of the shock front is recorded in each computational cell. The leading front at time $t$ is then extracted with a marching square algorithm [11]. The process and a grid convergence study are presented in [18]. 


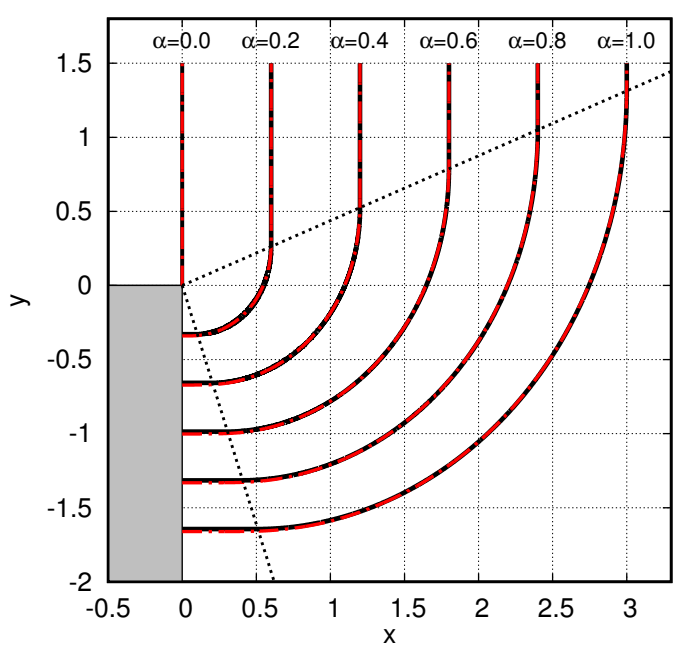

(a)

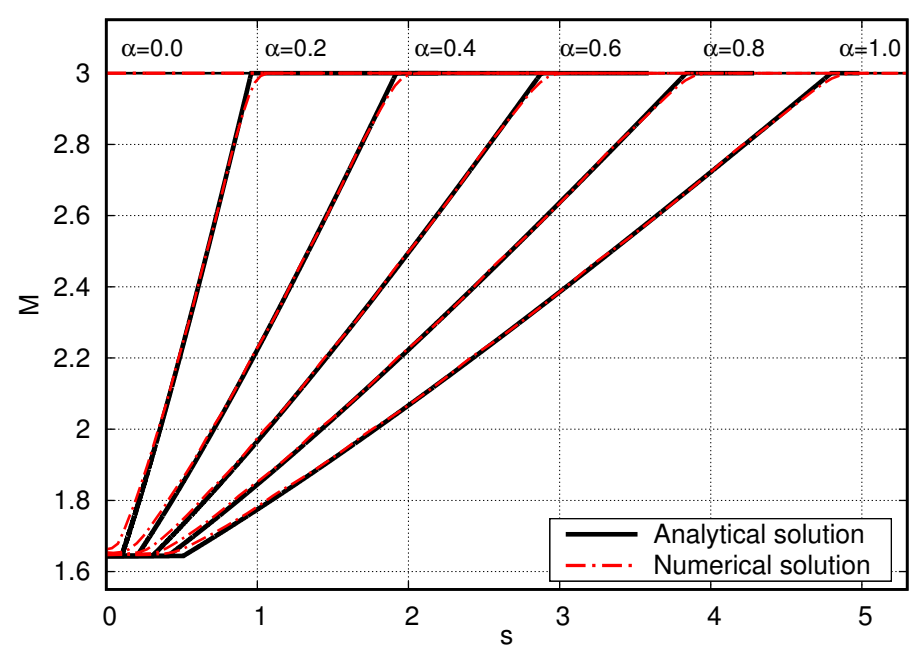

(b)

Figure 18: Diffraction of a planar shock at Mach number $M_{0}=3$ over a convex corner of angle $\theta_{\mathrm{w}}=-\pi / 2$. Comparison between the analytical solution (eqs. (19)-(20) and (33)) and the numerical solution for $\Delta s=0.0025$. Fig. (a): Successive shock positions. The dashed lines delimit the expansion zone. Fig. (b): Mach number along the shock.

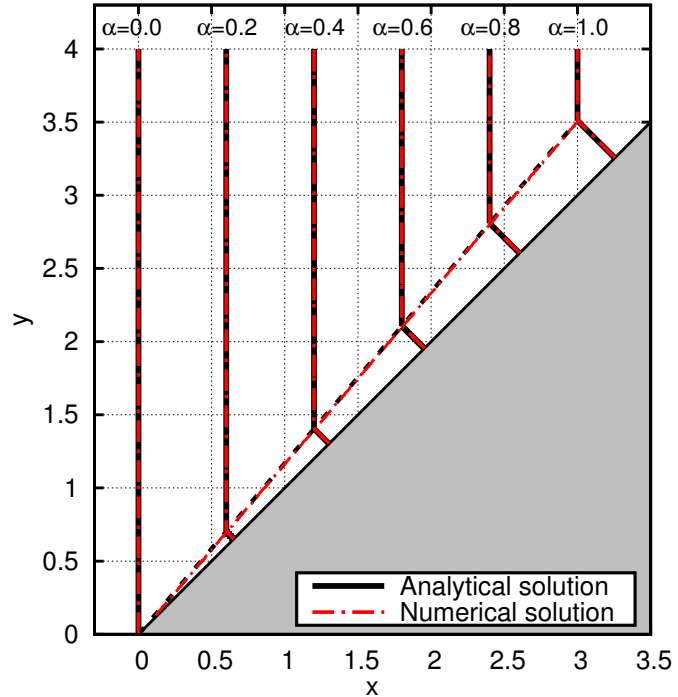

(a)

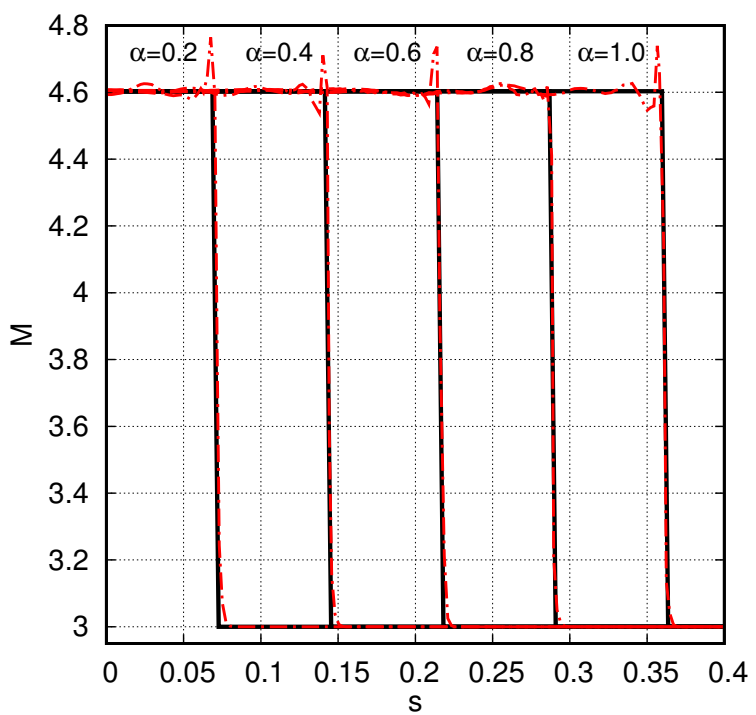

(b)

Figure 19: Diffraction of a planar shock at Mach number $M_{0}=3$ over a concave corner of angle $\theta_{\mathrm{w}}=\pi / 4$. Comparison between the analytical solution (21) and the numerical solution for $\Delta s=0.0025$. Fig. (a): Successive shock positions. Fig. (b): Mach number along the shock.

\subsection{Diffraction over a convex corner}

Let us consider the planar shock diffraction at Mach number $M_{0}$ over a convex corner of deflection angle $\theta_{\mathrm{w}}<0$ as presented in section 2.3. The solution is determined by (19)-(20) 


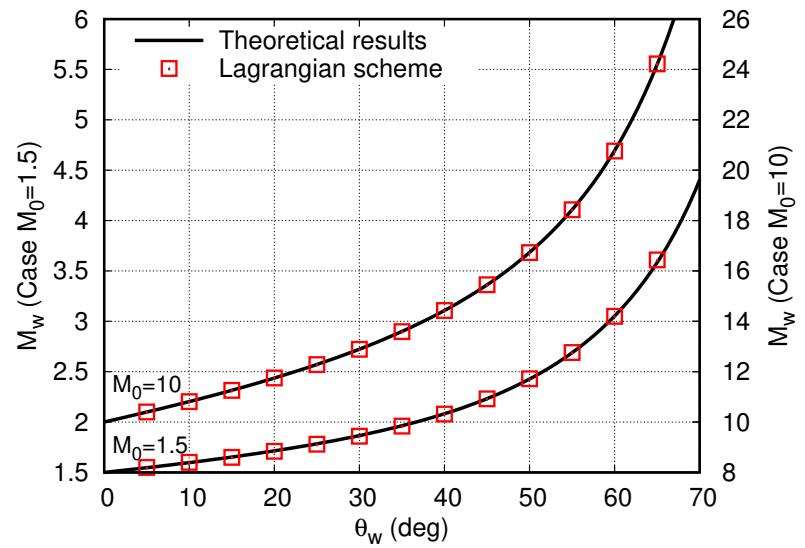

(a)

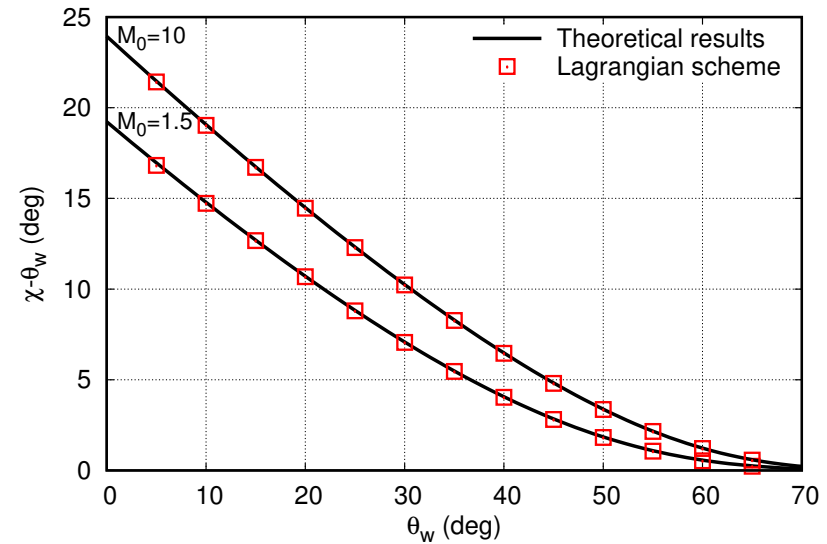

(b)

Figure 20: Results for the planar shock diffraction at Mach number $M_{0}=1.5$ and 10 over a concave corner for any deflecion angles $\theta_{\mathrm{w}}$. The analytical solution is defined by (21) and the numerical results are obtained for $\Delta s=0.01$. Fig. (a): Wall Mach number. Fig. (b): Direct angle between the $(O x)$ axis and the shock-shock trajectory.

with $A u(M)=\sqrt{\frac{M^{2}-1}{\lambda(M)}}$ for GSD (eq. (8)) and $A u(M)=k \frac{M^{2}-1}{2 M^{2}}$ for GSDT (eq. (33)).

Figure 21 displays the analytical solutions and the experimental data from [34] for $\theta_{\mathrm{w}}=$ $-\pi / 2$. We denote a better estimation of the shock front with GSDT, especially for weak shocks. At higher Mach numbers, one notes the existence of an inflection point on the front near the wall on experimental data as seen in figures 21(a) and 21(b). As detailed in [4], the post-shock effects, not taken into account in GSD and GSDT, are responsible for this behaviour.

In figure 22, the wall Mach number after diffraction is compared between GSD, GSDT and experimental data taken from [29]. Results for GSD and GSDT are obtained with relations (22) and (24)-(25) respectively. As expected, for $M_{0} \leq 2.5$ the GSDT model is in good agreement with experimental data contrary to Whitham's original model. A solution exists for all cases with the GSDT model. For stronger shocks and larger deflection angles $\left(-\theta_{w}>60^{\circ}\right)$ both models overestimate the wall Mach number. Notice that, for $M_{0}>2.5$, the post-shock flow has a significant influence over the shock propagation. The front is further slowed down near the wall resulting in the formation of an inflection point $[4,17]$. This phenomenon is not taken into account in the present models, which could explain, at least in part, the overestimation of the wall Mach numbers in comparison to experimental data.

\subsection{Combination of expansion waves}

Let us now study the planar shock diffraction at Mach number 2 over a rotated mound as experimented by Skews [30]. The initial shock evolves from the left to the right and diffracts over three successive convex corners. The solution is then a combination of expansion waves 


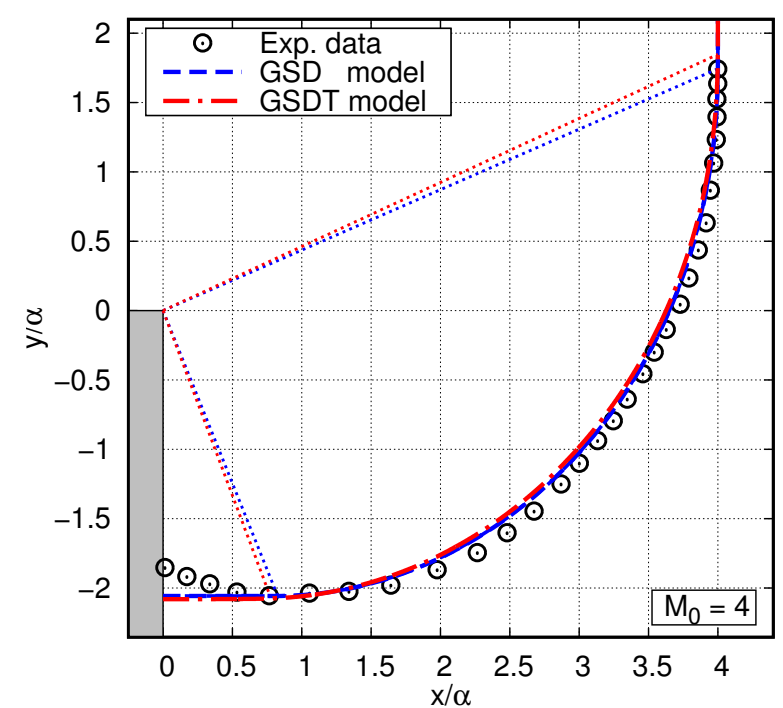

(a)

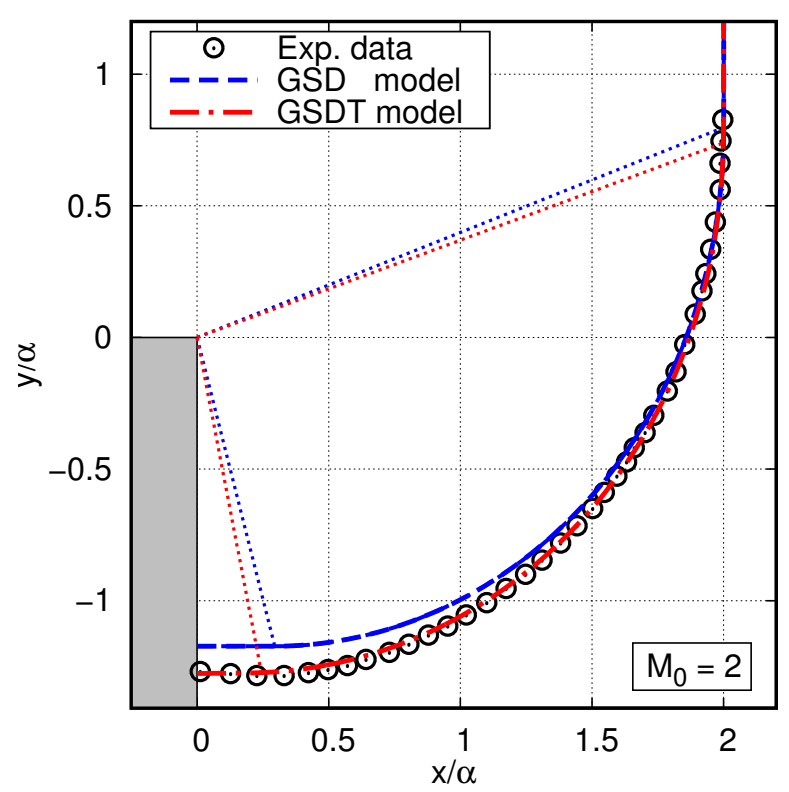

(c)

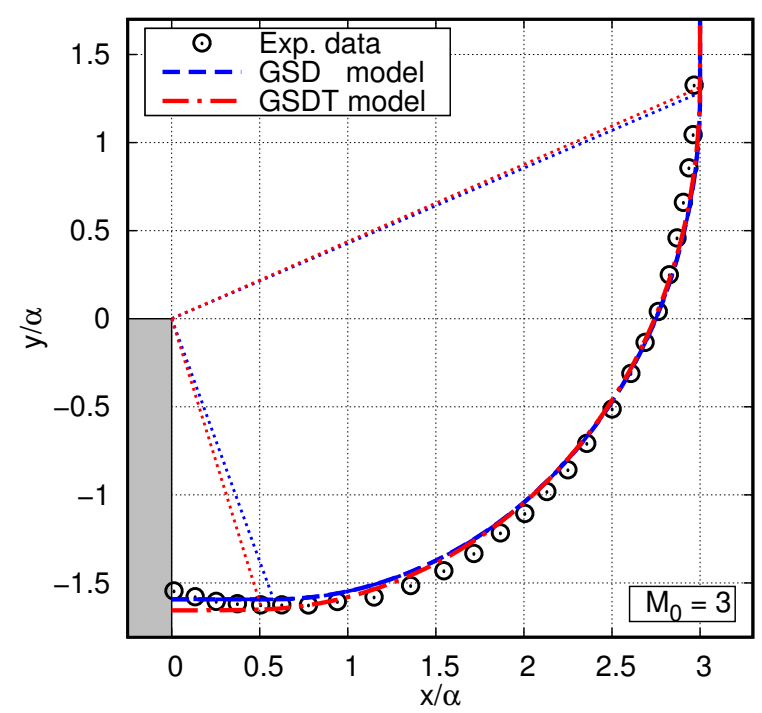

(b)

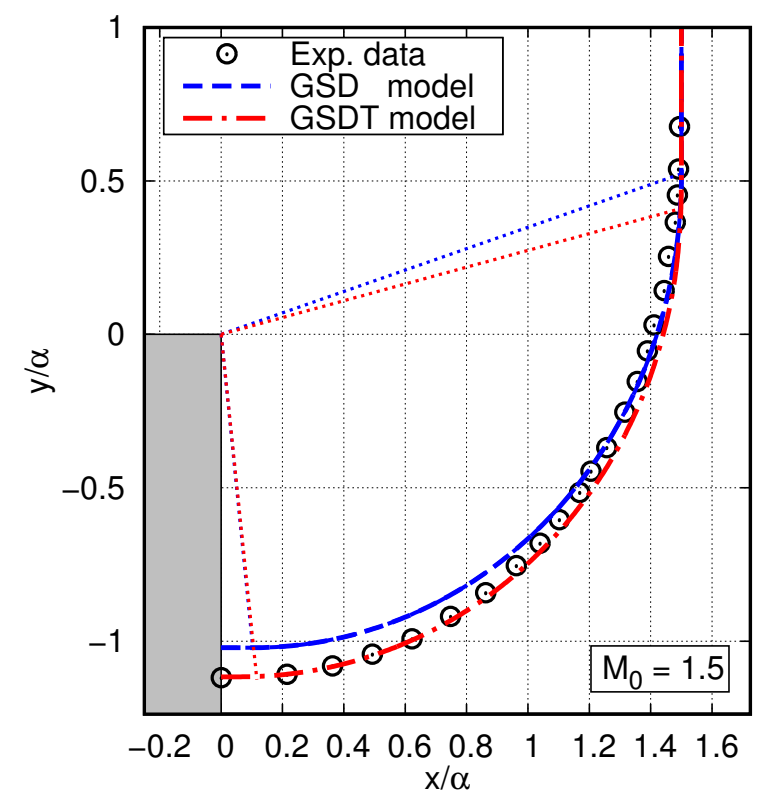

(d)

Figure 21: Diffraction of a planar shock at Mach number $M_{0}$ over a convex corner of angle $-90^{\circ}$. Experimental data taken from [34]. The solution is given by (19)-(20), where the velocity of the perturbations is defined by (8) for the GSD model and (33) for the GSDT model.

and requires the use of the Lagrangian scheme introduced in section 4 . The shock front positions at three different times are compared with experimental data from [30]. We observe that taking into account transverse flow significantly improves the solution near the wall. 

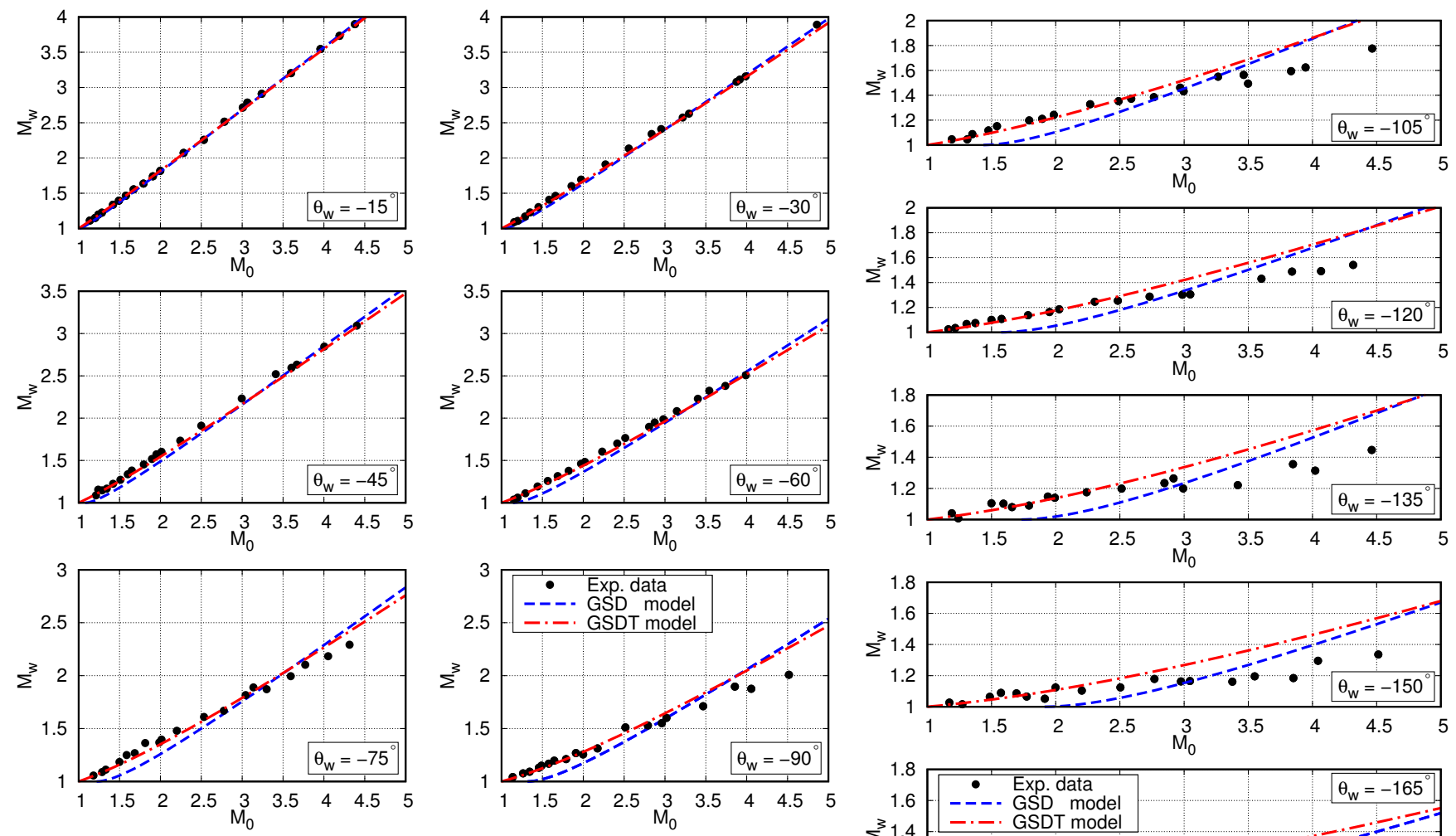

(a)
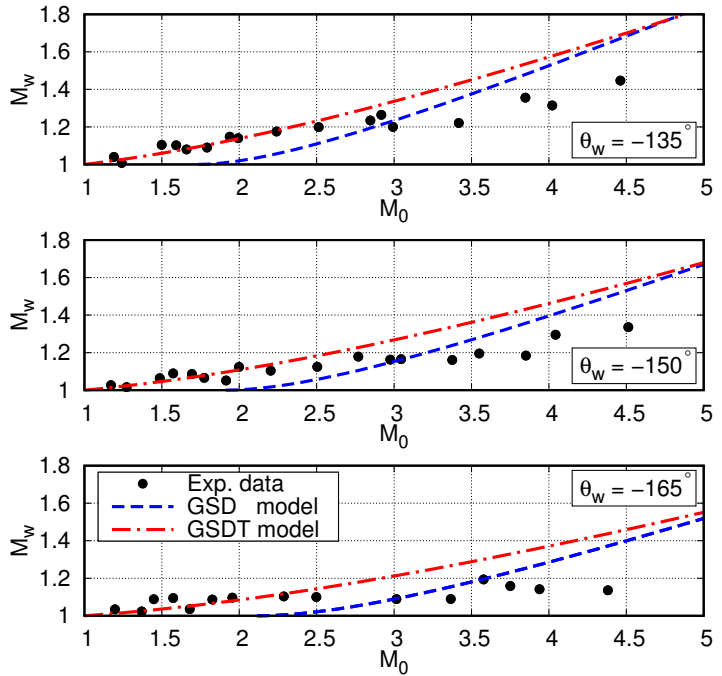

(b)

Figure 22: Diffraction of a planar shock at Mach number $M_{0}$ over a convex corner of angle $\theta_{\mathrm{w}}$. Wall Mach number after diffraction, $M_{\mathrm{w}}$, with respect to $M_{0}$, for different $\theta_{\mathrm{w}}$. Comparison between experimental data (black circles) from [29], the GSD model (22) (blue dashed curve), and the GSDT model (24)-(25) (red dash dotted curve).

\subsection{Diffraction over a mound}

To finish, let us consider a more complex problem for which the solution is a combination of expansion waves and shock-shocks. This problem is the planar shock diffraction at Mach number $M_{0}=4$ over a mound. The different faces of the mound and the ground behind it are numbered from 1 to 4 (see Fig. 24(a)). The shock interaction with the obstacle results in four successive diffractions. The problem is compressive on faces 1 and 4 leading to the formation of two shock-shocks. On the contrary, the shock is expansive when it reaches faces 2 and 3 resulting in expansion waves on the front.

For a strong shock, $M_{0}=4$ here, the shock is planar near the wall in such a way that each diffraction is locally similar to the elementary Riemann problems. Diffractions then correspond locally to planar shock diffraction over a concave corner of deflection angle $45^{\circ}$ on faces 1 and 4 , and a convex corner of deflection angle $-45^{\circ}$ for faces 2 and 3 . The wall Mach number, $M_{\mathrm{w}}$, can thus be estimated with (21a) for faces 1 and 4 for the GSD and 


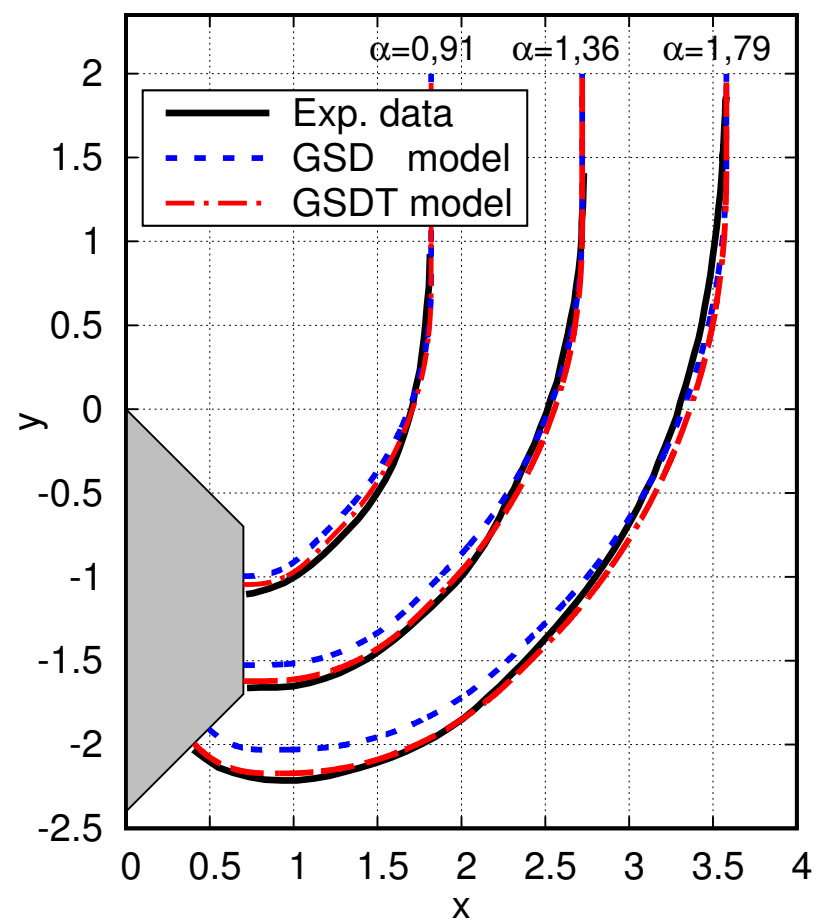

Figure 23: Diffraction of a planar shock at Mach number 2 over a rotated mound. The shock travels from the left to the right. Numerical simulation are obtained for $\Delta s=0.0025$. Experimental data are taken from [30].

GSDT models. For faces 2 and $3, M_{\mathrm{w}}$ is calculated with (22) for GSD and (24)-(25) for the GSDT model. The theoretical values determined with these relations are presented in table 1. For weaker values of $M_{0}$, the reflection on part 4 may become regular which is not

\begin{tabular}{|c|c|c|c|c|}
\hline$M_{\mathrm{w}} /$ Face & 1 & 2 & 3 & 4 \\
\hline GSD & 6.158 & 4.361 & 3.102 & 4.762 \\
\hline GSDT & 6.158 & 4.247 & 2.976 & 4.567 \\
\hline
\end{tabular}

Table 1: Theoretical wall Mach numbers for GSD and GSDT models after diffraction on each face for the planar shock diffraction at Mach number $M_{0}=4$ over the mound shown in figure 24(a)

compatible with Whitham's theory where a Mach stem is always present, and explains our choice.

As compressive problems are not affected for the GSDT model, results on face 1 are similar to GSD. This is no longer the case when the shock interacts with face 2, the front being expansive. The modification of GSD results in a slightly smaller value of $M_{\mathrm{w}}$ in comparison to the original model. This difference is more pronounced for the diffraction over face 3 as the incoming shock is weaker for GSDT than for GSD (Mach 4.247 instead of Mach 4.361, see table 1). Finally, the Mach stem behind the mound is slowed down for 


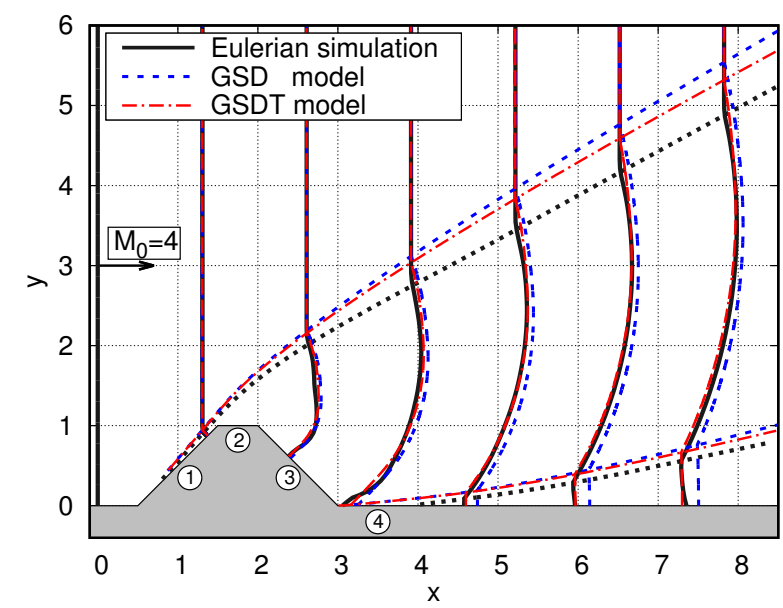

(a)



(b)

Figure 24: Diffraction of a planar shock at Mach number $M_{0}=4$ over a mound. Fig. (a): Successive shock positions. Fig. (b): Wall Mach number. Numerical simulation with Lagrangian scheme: $\Delta s=0.0025, d_{c}=0.05$, Eulerian simulation: $\Delta x=\Delta y=0.00125$.

GSDT in comparison with GSD. This differences lead to solutions which significantly differ between both models on face 4 . Successive numerical shock positions and wall Mach number are presented in figure 24. In this example, note the improved agreement between the GSDT model and Eulerian results. The Eulerian simulation is performed with a cell size of 1/800 to achieve grid convergence. Here, the post-shock flow has a discernable influence on the leading shock due to a double Mach reflection [2]. Oscillations of the Mach number are observed and it gets difficult to extract the velocity of the shock as seen in figure 24(b). The duration of these simulations is a few hours for the Eulerian case and only a few minutes with the GSDT model.

\section{Conclusion and perspectives}

In this paper, an ad-hoc extension of the GSD model to transverse flow is presented in order to remove its limitation for expansive shocks. The correction is only active in expansive regions of the front and does not change the hyperbolicity of the model. This new approach is called GSDT, T standing for Transverse. For expansive cases considered here, results with GSDT are in better agreement with experimental data. The modification of expansion waves in the GSD model has an influence on the solution for more complex problems as shown for the interaction of a planar shock over a mound. For the cases presently treated in the paper, the GSDT model gives better results than GSD compared to Eulerian simulation or experimental results.

The new 2D conservative Lagrangian algorithm inspired from Henshaw et al. [7] has been detailed and fully validated. In the original scheme, the $A-M$ relation is integrated from the initial time to ensure the conservativity of the scheme. Our main contribution is its step 
by step integration written with an additional general term in the $A-M$ relation. This allows an easy integration of the transverse term, or any source term. Let us underline that the resolution of GSD for inhomogeneous but at rest media is now direct with this algorithm, contrary to the original one [21].

In the future, GSD and GSDT models may be compared on more complex configurations. A monotone algorithm should also be developed in order to avoid oscillations which complicate the estimation of the local curvature of the shock. At last, it is worth mentioning that the GSD model, and so the GSDT model, always restitute a Mach stem for compressive problems. Some additional mechanisms should be considered to account for regular reflection, especially for weak shocks. These issues could be addressed in further investigations.

\section{Acknowledgements}

Part of this work has been possible thanks to the LETMA collaboration: a contractual research laboratory between CEA, CNRS, École Centrale Lyon, C-Innov and Sorbonne Université. We thank Pr. Schwendeman, from Rensselaer Polytechnic Institute Troy NY USA, for useful discussions about the Lagrangian scheme.

\section{References}

[1] T.V. Bazhenova, L.G. Gvozdeva, and Y.V. Zhilin. Change in the shape of the diffracting shock wave at a convex corner. Acta Astronautica, 6(3):401-412, 1979.

[2] G. Ben-Dor. Handbook of Shock Waves, volume 2, chapter 8.1: Oblique Shock Wave Reflections, pages 68-179. Academic Press, 2000.

[3] C. Besset and E. Blanc. Propagation of vertical shock waves in the atmosphere. The Journal of the Acoustical Society of America, 95(4):1830-1839, 1994.

[4] J.P. Best. A generalisation of the theory of geometrical shock dynamics. Shock Waves, 1(4):251-273, 1991.

[5] C.J. Catherasoo and B. Sturtevant. Shock dynamics in non-uniform media. Journal of Fluid Mechanics, 127:539-561, 1983.

[6] S. Gottlieb and C.W. Shu. Total variation diminishing Runge-Kutta schemes. Mathematics of computation of the American Mathematical Society, 67(221):73-85, 1998.

[7] W.D. Henshaw, N.F. Smyth, and D.W. Schwendeman. Numerical shock propagation using geometrical shock dynamics. Journal of Fluid Mechanics, 171:519-545, 1986.

[8] H.T. Huynh. Accurate monotone cubic interpolation. SIAM Journal on Numerical Analysis, 30(1):57-100, 1993. 
[9] H. Jourdren. HERA: a hydrodynamic AMR platform for multi-physics simulations. In Adaptive Mesh Refinement-Theory and Applications, pages 283-294. Springer, 2005.

[10] B.I. Kvasov. Monotone and convex interpolation by weighted quadratic splines. Advances in Computational Mathematics, 40(1):91-116, 2014.

[11] W.E. Lorensen and H.E. Cline. Marching cubes: A high resolution 3d surface construction algorithm. ACM siggraph computer graphics, 21(4):163-169, 1987.

[12] Y. Noumir, A. Le Guilcher, N. Lardjane, R. Monneau, and A. Sarrazin. A fast-marching like algorithm for geometrical shock dynamics. Journal of Computational Physics, 284:206-229, 2015.

[13] S. Osher and C.W. Shu. High-order essentially nonoscillatory schemes for HamiltonJacobi equations. SIAM Journal on Numerical Analysis, 28(4):907-922, 1991.

[14] K. Oshima. Propagation of spacially non-uniform shock waves. ISAS report, 30(6):195, 1965.

[15] K. Oshima, K. Sugaya, M. Yamamoto, and T. Totoki. Diffraction of a plane shock wave around a corner. ISAS report, 30(2):51-82, 1965.

[16] M. Pandey and V.D. Sharma. Kinematics of a shock wave of arbitrary strength in a non-ideal gas. Quarterly of Applied Mathematics, 67(3):401-418, 2009.

[17] J. Ridoux. Contribution au développement d'une méthode de calcul rapide de propagation des ondes de souffle en présence d'obstacles. PhD thesis, Université Pierre et Marie Curie, 2017.

[18] J. Ridoux, N. Lardjane, L. Monasse, and F. Coulouvrat. Comparison of Geometrical Shock Dynamics and Kinematic models for shock wave propagation. Shock Waves, 27(5):1-16, 2017.

[19] P. Romon. Introduction à la géométrie différentielle discrète. Ellipses, 2013.

[20] D.W. Schwendeman. A numerical scheme for shock propagation in three dimensions. Proceedings of the Royal Society of London A: Mathematical, Physical and Engineering Sciences, 416(1850):179-198, 1988.

[21] D.W. Schwendeman. Numerical shock propagation in non-uniform media. Journal of Fluid Mechanics, 188:383-410, 1988.

[22] D.W. Schwendeman. A new numerical method for shock wave propagation based on geometrical shock dynamics. Proceedings of the Royal Society of London A: Mathematical, Physical and Engineering Sciences, 441(1912):331-341, 1993. 
[23] D.W. Schwendeman. A higher-order Godunov method for the hyperbolic equations modelling shock dynamics. Proceedings of the Royal Society of London A: Mathematical, Physical and Engineering Sciences, 455(1984):1215-1233, 1999.

[24] D.W. Schwendeman. On converging shock waves of spherical and polyhedral form. Journal of Fluid Mechanics, 454:365-386, 2002.

[25] D.W. Schwendeman and G.B. Whitham. On converging shock waves. Proceedings of the Royal Society of London A: Mathematical, Physical and Engineering Sciences, 413(1845):297-311, 1987.

[26] V.D. Sharma and C. Radha. On one-dimensional planar and nonplanar shock waves in a relaxing gas. Physics of Fluids, 6(6):2177-2190, 1994.

[27] V.D. Sharma and C. Radha. Three dimensional shock wave propagation in an ideal gas. International Journal of Non-Linear Mechanics, 30(3):305-322, 1995.

[28] W. Shi and C.K. Cheung. Performance evaluation of line simplification algorithms for vector generalization. The Cartographic Journal, 43(1):27-44, 2006.

[29] B.W. Skews. The shape of a diffracting shock wave. Journal of Fluid Mechanics, 29(02):297-304, 1967.

[30] B.W. Skews. Shock wave diffraction on multi-facetted and curved walls. Shock Waves, 14(3):137-146, 2005.

[31] D.A. Tariq, J.B. Bdzil, and D.S. Stewart. Level set methods applied to modeling detonation shock dynamics. Journal of Computational Physics, 126(2):390-409, 1996.

[32] G.B. Whitham. A new approach to problems of shock dynamics. Part I: Twodimensional problems. Journal of Fluid Mechanics, 2(2):145-171, 1957.

[33] G.B. Whitham. A new approach to problems of shock dynamics. Part II: Threedimensional problems. Journal of Fluid Mechanics, 5(3):369-386, 1959.

[34] G.B. Whitham. Linear and Nonlinear Waves, chapter 8: Shock Dynamics. John Wiley \& Sons, Inc., 3rd edition, 1999. 